\title{
ON THE APPLICATION OF VARIATIONAL THEORY TO URBAN NETWORKS
}

\author{
A Preprint
}

Gabriel Tilg*

Chair of Traffic Engineering and Control

Department of Civil, Geo and Environmental Engineering

Technical University of Munich, Germany

\author{
Lukas Ambühl \\ Traffic Engineering Group, Institute for Transport Planning and Systems \\ ETH Zurich, Switzerland
}

Sergio Batista

Division of Engineering

New York University Abu Dhabi, United Arab Emirates

Fritz Busch

Chair of Traffic Engineering and Control

Department of Civil, Geo and Environmental Engineering

Technical University of Munich, Germany

Monica Menendez

Division of Engineering

New York University Abu Dhabi, United Arab Emirates

July 25,2020

\begin{abstract}
The well-known Lighthill-Whitham-Richards (LWR) theory is the fundamental pillar for most macroscopic traffic models. In the past, many methods were developed to numerically derive solutions for LWR problems. Examples for such numerical solution schemes are the cell transmission model, the link transmission model, and the variational theory (VT) of traffic flow. So far, the latter framework found applications in the fields of traffic modelling, macroscopic fundamental diagram estimation, multi-modal traffic analyses, and data fusion. However, these studies apply VT only at the link or corridor level. To the best of our knowledge, there is no methodology yet to apply VT at the network level. We address this gap by developing a VT-based framework applicable to networks. Our model allows us to account for source terms (e.g. inflows and outflows at intersections) and the propagation of spillbacks between adjacent corridors consistent with kinematic wave theory. We show that the trajectories extracted from a microscopic simulation fit the predicted traffic states from our model for a simple intersection with both source terms and spillbacks. We also use this simple example to illustrate the accuracy of the proposed model. Additionally, we apply our model to the Sioux Falls network and again compare the results to those from a microscopic simulation. Our results indicate a close fit of traffic states, but with substantially
\end{abstract}

*Corresponding author: gabriel.tilg@tum.de. 
A PREPRINT - JULY 25, 2020

lower computational cost. The developed methodology is useful for network-wide traffic state estimations in real-time, or other applications within a model-based optimization framework.

Keywords variational theory $\cdot$ network modeling $\cdot$ kinematic wave theory $\cdot$ LWR model $\cdot$ traffic flow theory

\section{Introduction}

The Lighthill-Whitham-Richards (LWR) theory (Lighthill and Whitham, 1955; Richards, 1956) is the base for most macroscopic models of traffic dynamics at the link level. The first-order model relates the conservation law (see eq.(1)) and the fundamental diagram (FD) $q=Q(k)$ of traffic:

$$
\frac{\partial k}{\partial t}+\frac{\partial q}{\partial x}=0
$$

where $q$ is the flow in vehicles per hour, $k$ is the density in vehicles per kilometer, and $t, x$ represent the time and space coordinates, respectively. Newell (1993) formulated the LWR theory in terms of the cumulative vehicle count $N(x, t)$ in his simplified kinematic wave theory (KWT). It describes the number of vehicles $N$ that have passed point $x$ by time $t$. The cumulative count $N$ across all $(x, t)$ forms a surface which is also known as the Moskowitz function (Moskowitz, 1965). The derivatives of this surface at a given point $(x, t)$ are the flow $q(x, t)$ and the density $k(x, t)$. Newell (1993) formulated the problem as follows:

$$
\frac{\partial N}{\partial t}-Q\left(-\frac{\partial N}{\partial x}\right)=0
$$

The formulation of the LWR in terms of $N(x, t)$ corresponds to a partial differential equation of the HamiltonJacobi type. Over the past decades numerous approaches have been developed to numerically solve the LWR partial differential equation. Generally, the equation can be represented in three different coordinate systems (Laval and Leclercq, 2013): eulerian $(t, x)$, lagrangian $(t, N)$, and in $(N, x)$. Numerical schemes exist for two of these systems. In the eulerian representation, popular methods are the cell transmission model (CTM) (Daganzo, 1992, 1995), the link transmission model (LTM) (Yperman, 2007; Tampère et al., 2011; Han et al., 2015; Jin, 2015), techniques from partial differential equations theory such as the grid-free method by Mazaré et al. (2011), and the variational theory (VT) of traffic flow (Daganzo, 2005a,b; Daganzo and Menendez, 2005; Claudel and Bayen, 2010a,b). Several works have also explored the lagrangian space for solving LWR partial differential equations (e.g. Leclercq et al., 2007). For a more detailed overview on numerical schemes to solve KWT problems, we refer the reader to Seo et al. (2017).

The comparison of VT to other solution methods such as the CTM or the LTM reveals several advantages. The CTM is known to exhibit numerical viscosity in which shock waves are inaccurately represented as smooth variations. These numerical errors converge to zero with decreasing time-step sizes. The solution provided by VT is exact for piece-wise linear flow-density FDs independent of the time-step size. While such numerical error is small for the LTM as well, the latter does not allow to explicitly consider complex heterogeneous KWT problems. Such problems might include space-time dependent FDs, in combination with any type of stationary and/or moving bottlenecks. In contrast, VT permits the evaluation of KWT problems including heterogeneities in time and space (Daganzo and Menendez, 2005). These aspects distinguish VT from the CTM and the LTM, and underline its superiority at the link level. Thus, we focus on VT and propose a framework to further extent its applicability to the network level.

The concept of VT has been applied in several contexts so far, with a few studies advancing the existing VT modelling techniques. Friesz et al. (2013) developed a dynamic user equilibrium framework for networks. Traffic dynamics were modeled based on the LWR theory, and numerically represented with VT. Yet, they did not consider spillbacks across intersections in their study, and thus underestimated delays for heavily congested scenarios. Similarly, Li and Zhang (2015) described the performance of queuing systems with multiple sequential and parallel bottlenecks based on VT. However, their framework did not account for spillover effects. Chow et al. (2015) utilized the ability of VT to model complex traffic dynamics at the corridor level on urban arterials in London (UK) and compared it to CTM. They confirmed the high-quality results of VT for such settings, especially for platoon dispersion and moving bottlenecks. Hans et al. (2015) developed a mesoscopic model based on VT to exactly estimate travel times for urban arterials. While the application of VT was still limited to the link and corridor levels, these studies show that VT has been recognized as a powerful numerical scheme for solving KWT problems. 
VT is not restricted to the study of car traffic only. It has also been utilized for multi-modal traffic analysis. Guler et al. (2016) investigated innovative transit signal priority designs and estimated its effects on intersection performance with VT. Similarly, Wu et al. (2017) analyzed the impacts of bus stop locations and TSP on intersection operations. Gayah et al. (2016) studied the impacts of general obstructions on the capacity of an isolated intersection, and computed the capacity losses using VT. Saeednia and Menendez (2016) evaluated the effect of truck platoons on freeway traffic also using VT.

Additionally, VT has been applied for the approximation of the macroscopic fundamental diagram (MFD). The method of cuts introduced by Daganzo and Geroliminis (2008) estimates the MFD for a homogeneous ring road based on VT. Accordingly, related extensions and studies also refer and exploit VT (Boyaci and Geroliminis, 2011; Leclercq and Geroliminis, 2013; Laval and Castrillón, 2015; Tilg et al., 2020; Daganzo and Knoop, 2016; Leclercq and Paipuri, 2019; Girault et al., 2016; Ambühl et al., 2018; Loder et al., 2019). Again, these approximation methods apply VT only at the corridor level, even when trying to estimate traffic conditions at the network level.

Another field of application is traffic state estimation and data fusion (Mehran et al., 2012; Sun and Ban, 2013). This line of research has explored the application of VT to reconstruct vehicle trajectories based on data from fixed and/or mobile sensors, as well as data fusion algorithms. Mehran and Kuwahara (2013) further extended this approach to predict vehicle trajectories based on real-time and historical data. Duret and Yuan (2017) proposed a traffic state estimation framework based on eulerian and lagrangian observations. Their data fusion framework includes the concept of VT. More works on developing data fusion frameworks exploiting the concept of VT concern real-time applications (Kawasaki et al., 2017) and measurements from vehicles running on opposite lanes (Kawai et al., 2019; Takenouchi et al., 2019).

Not only has VT been applied in several contexts, but a number of attempts to extend the original framework have also been reported. For example, Lebacque and Khoshyaran (2013) showed that generic second-order models admit a Hamilton-Jacobi and variational formulation as an optimal control problem. Costeseque and Lebacque (2014) numerically investigated the VT formulation for higher order traffic models. Additionally, the deterministic nature of VT was questioned and by the inclusion of stochastic shortest path algorithms new fields of application were made accessible. Wada et al. (2018) applied VT for coordinated traffic signal control for both deterministic and stochastic demands. Dakic et al. (2020) applied a stochastic shortest path search within VT, to estimate the capacity of bi-modal corridors. Laval et al. (2016) explored the impact of source terms on VT. They developed a framework to consider continuous inflows and outflows (i.e. source terms) which could represent trips starting and ending throughout the link.

The investigation of the related literature indicates the potential of VT for traffic modelling, MFD estimation, data fusion, and other methodological extensions. However, to the best of our knowledge, none of the previous studies have started to explore the application of VT at the network level accounting for its complexities. To address this gap, we propose a VT extension that allows us to model the traffic dynamics at the network level. The contributions are fourfold. First, we include inflows and outflows in the mathematical formulation of VT. Contrary to Laval et al. (2016), these source terms accept any values from the set of real numbers, most importantly, they also include zero. Second, we model the propagation of spillbacks across the network. This allows us to model the evolution of network-wide congestion patterns. Third, we evaluate the proposed methodology and compare it with a microscopic KWT model implemented in SUMO (Lopez et al., 2018). This enables us to show a proof of our concept, and to investigate the model's accuracy. Fourth, we propose an algorithm to apply our model to any road network, and show its applicability using the well-known example of the Sioux Falls network.

This paper is organized as follows. Section 2 provides a brief background of VT. Section 3 proposes a VT-based methodology to estimate traffic state propagation throughout a network. This includes the consideration of inflows and outflows at intersections and the correct propagation of spillbacks. Section 4 provides a proof of concept and evaluates the accuracy of the proposed framework for small networks. Section 5 demonstrates the applicability of our methodology for the case of the Sioux Falls network. Section 6 highlights the conclusion of this study and outlines potential future research topics.

\section{Background}

Daganzo (2005a) formulated the VT framework the first time to determine the cumulative number of vehicles, i.e. the Moskowitz function, $N(x, t)$ which have passed the location $x$ of a road by time $t$ for given boundary conditions. The surface formed by $N$ across all $(x, t)$ is continuous but not differentiable at shockwaves under the assumption of flow conservation. Figure 1 illustrates the basic concept of VT. 
A PREPRINT - JuLY 25, 2020

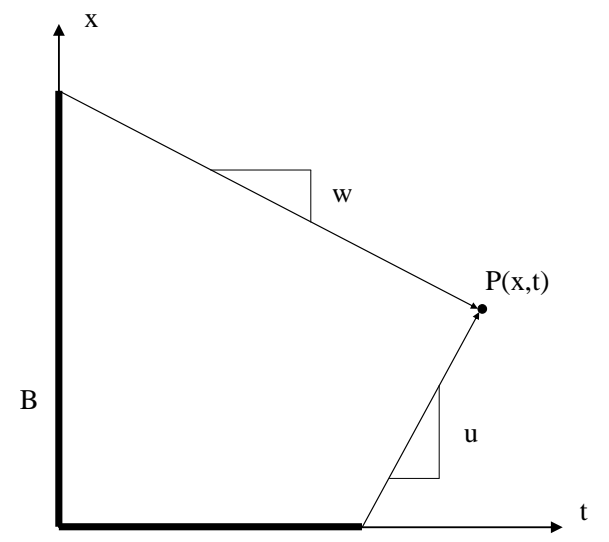

Figure 1: Concept of VT.

A necessary input for solving a KWT problem is an FD, i.e. $Q(k)$. Such an FD is characterized by the free-flow speed $u$, the backward wave speed $w$, and the jam density $\kappa$. The capacity is derived at the critical density, i.e. $Q\left(k_{c}\right)$. Additionally, boundary data $N_{B}$ along a curve $B$ are required, as illustrated by a thick black line in Figure 1. Let us now discuss, how to determine $N$ at point $P(x, t)$. We define a set of valid paths $p \in \mathcal{P}$, that start at the boundary $B$ and end at $P$. A path $p$ is valid if its slope ranges between the extremal speeds $v \in[w, u]$. Related to the slope, each path $p$ has a certain cost $c_{p}$, corresponding to the maximum traffic rate that can pass a moving observer traveling along that path. Given the set $\mathcal{P}$ with costs $c_{p}$ for all paths $p \in \mathcal{P}$, and the boundary value $N_{B, p}$ associated to the path $p$, the cumulative count $N_{P}$ is found as follows:

$$
N_{P}=\min _{p \in \mathcal{P}}\left\{N_{B, p}+c_{p}\right\} .
$$

Assuming a triangular FD further simplifies the procedure. In this case, the formulation becomes exact (Daganzo, 2005a). We also define $\theta=\frac{u}{w}$ as the ratio of the free-flow and the backward wave speed. If $\theta$ is an integer, the so-called lopsided variational graph can be constructed (see Daganzo and Menendez, 2005). This graph represents a discretized grid, where $\Delta t$ and $\Delta x=u \Delta t$ are the spatial and temporal grid lengths, respectively. We denote the variational graph as $G(\mathcal{C}, \mathcal{I})$, where $\mathcal{C}$ is the set of corridors, and $\mathcal{I}$ is the set of intersections. For the original VT formulation, $|\mathcal{C}|=1$. The case of a multi-dimensional graph will be explained in the following sections. One can then solve a KWT problem to determine $N$ on each node of $G$, by conducting a shortest-path search starting at the boundary. In the absence of moving bottlenecks, the necessary paths to consider become straight lines and have either extremal slopes, or slopes equal to zero at signals, i.e. $v \in\{w, 0, u\}$. We can find $N(x, t)$ for this case as the minimum of the shortest paths coming from three nodes, one upstream node at $x-\Delta x$, one downstream node at $x+\Delta x$, and one node at the same location $x$ (Daganzo and Menendez, 2005; Leclercq and Paipuri, 2019):

$$
\begin{aligned}
N(x, t)=\min & (N(x-\Delta x, t-\Delta t), \\
& N(x+\Delta x, t-\theta \Delta t)+\Delta x \kappa, \\
& N(x, t-\Delta t)+\beta) .
\end{aligned}
$$

The first term in equation (4) refers to free-flow states travelling from upstream, the second one to congested states travelling from downstream, and the third term accounts for bottleneck induced capacity constraints at the same location as the target node. For free-flow states the cost of the edge, i.e. the maximum rate at which traffic can pass a moving observer travelling at a speed corresponding to the slope of that edge, is zero. For congested states the cost of the edge is $\Delta x \kappa$. For capacity constraints at the same location the cost of the edge is $\beta$. If a signal at position $x$ is red during the interval $[t-\Delta t, t], \beta=0$. Otherwise, $\beta=Q\left(k_{c}\right)$, i.e. the link capacity. This formulation allows to apply VT to any signalized or unsignalized corridor with a triangular FD, where $\theta$ is integer.

Figure 2 represents an excerpt of the variational graph $G$ which allows for a graphical interpretation of eq. (4). The graph $G$ is represented as a numerical grid with $\Delta x$ in the spatial and $\Delta t$ in the temporal dimension. For this figure, we set $\theta=2$. Note that VT is valid for any integer value of $\theta$. Additionally, the figure shows the 
three "from nodes": upstream, at the same location, and downstream of $P(x, t)$, as well as the corresponding paths along which the costs are calculated.

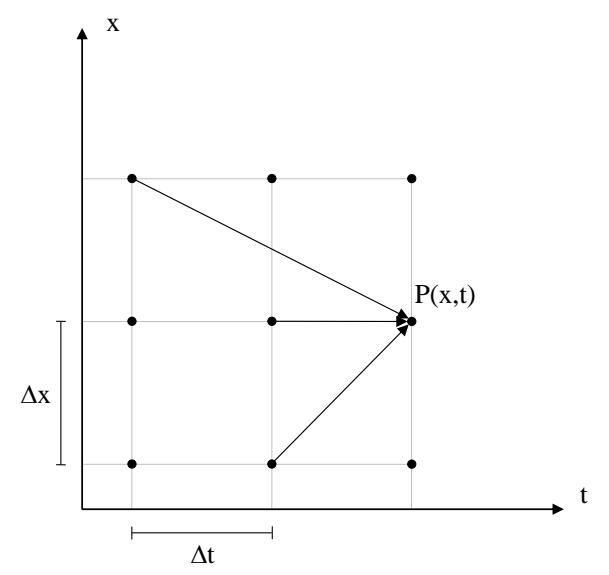

Figure 2: Excerpt of the variational graph.

Eq. (4) implies there are no inflows nor outflows within the corridor. This is because the occurrence of such source terms violates flow conservation along the corridor, which is the main assumption of the conservation equation (see eq.(1)). While the assumption of zero net inflows might hold for singular corridors, it represents an issue for general networks. Laval et al. (2016) studied the incorporation of such source terms into the VT framework. They provided a method to consider source terms when they are piece-wise continuous in space and time. However, their method is not able to handle source terms at intersections, as they are discrete in space and time, and include non-zero values during green and values equal to zero during red phases.

In the next section, we provide a framework to account for source terms in VT at intersections and model spillbacks. This constitutes the first building block to apply the VT-based framework at the network level. Hereafter, we refer to our framework as 'nVT' as abbreviation for network VT, and 'original VT' to the formulation introduced by Daganzo (2005a).

\section{Generalizing the VT framework to networks}

In this paper, we modify VT and establish a comprehensive model that allows to numerically solve complex heterogeneous KWT problems at the network level. We limit our networks to combinations of signalized corridors without partially conflicting traffic streams. Therefore, the focus lies on networks with signalized intersections with dedicated phases for the conflicting traffic streams. The relaxation of this assumption will be discussed later in Section 6. Below, we describe the overall framework which consists of three steps.

Step 0 initializes the problem by defining the infrastructure and the demand. This consists of the definition of the network topology, the signal control settings, and the temporal and spatial demand patterns. The latter aspect includes the origins as well as turning ratios at each intersection. Note that this implies an indirect definition of destinations. This allows to generate the network $\mathcal{N}$ and the data at the boundaries.

Step 1 involves the decomposition of the network $\mathcal{N}$ into a set of corridors $C \in \mathcal{C}$. This enables us to define a multi-dimensional variational graph in order to solve the given KWT problem. The graph has $(|\mathcal{C}|+1)$ dimensions, consisting of the corridors in $\mathcal{C}$, and one temporal dimension. We provide more details on this step in subsection 3.1.

Step 2 applies our VT framework, the $\mathrm{nVT}$, taking the multi-dimensional variational graph as input. Our framework builds an extension that incorporates source terms into the original VT concept. We treat turning flows as source terms at the location of intersections which are discrete in time and space. Moreover, the model replicates spillbacks across intersections in the network. This step is explained in detail in subsection 3.2 .

\subsection{Step 1: Decomposition of networks into corridors}

The problem initialization results in a network $\mathcal{N}$ consisting of intersections $I \in \mathcal{I}$ and links $L \in \mathcal{L}$. Additionally, we specify signal control settings, i.e. red and green times, and turning ratios $\alpha \in A$ at each 
intersection $I \in \mathcal{I}$, as well as origin flows. As discussed above, the definition of the variational graph requires the decomposition of the network $\mathcal{N}$ into a set of corridors $\mathcal{C}$. Thereby, the topological order including turning ratios $\alpha_{i j}$ between each pair of corridors $C_{i}$ and $C_{j}$ as well as control settings for $I \in \mathcal{I}$ have to be stored.

There are two main requirements for decomposing the network $\mathcal{N}$ into a variational graph:

1. The set $\mathcal{C}$ includes all links $L \in \mathcal{L}$.

2. Each link $L$ exists only once in $\mathcal{C}$, i.e. the corridors do not have any overlapping segments.

For small toy networks, we can determine the set $\mathcal{C}$ manually from $\mathcal{N}$. In other cases, this decomposition can be performed according to the actual layout of roads in the real network. This includes arterials, avenues and streets. Each road can be represented as a corridor $C$ and as such be incorporated in $G$. This will always satisfy both conditions mentioned above. Note that the resulting decomposed network, i.e. the set $\mathcal{C}$, will not affect the result. The KWT solution is determined by the demand (origin flows and turning ratios), as well as the supply (link FDs, link lengths, etc.). As these parameters are not affected by assigning links $L$ to corridors $C$, the KWT and therefore the VT solution are unaffected as well. In other words, the KWT solution is independent of the decomposition method.

Figure 3 schematically illustrates the process with an example. Figure 3 a depicts a network $\mathcal{N}$ consisting of five intersections, $\mathcal{I}=\left\{I_{1}, I_{2}, I_{3}, I_{4}, I_{5}\right\}$, and six unidirectional links, $\mathcal{L}=\left\{L_{1}, L_{2}, L_{3}, L_{4}, L_{5}, L_{6}\right\}$. Note that our method is not limited to unidirectional links, but this assumption simplifies the example. The intersections connecting the different corridors play an important role, as they serve as interfaces where flow is transferred from one corridor to the other, i.e. where source terms apply. We denote such intersections as inter-corridor connections to distinguish them from intersections without any turning flows (e.g. pedestrian crossings). For our example, we assume that $\alpha>0$ for all intersections. That is, they are all inter-corridor connections.

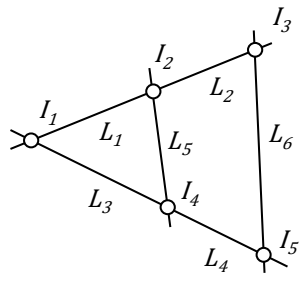

a) Network $\mathcal{N}$.

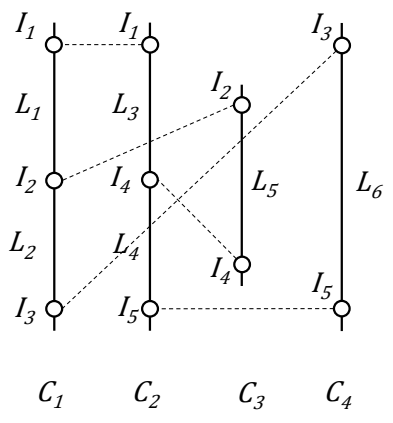

b) Set of corridors $\mathcal{C}$.

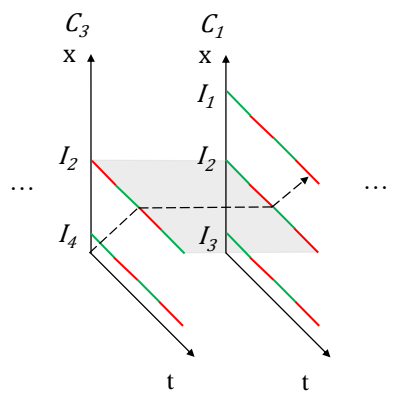

c) $(|\mathcal{C}|+1)$-dimensional variational graph.

Figure 3: Schematic illustration of the network decomposition and definition of a multi-dimensional variational graph.

We manually decompose $\mathcal{N}$ into a set $\mathcal{C}=\left\{C_{1}, C_{2}, C_{3}, C_{4}\right\}$ as shown in Figure $3 \mathrm{~b}$. This set contains only non-overlapping corridors covering the whole network. Both requirements stated above are then fulfilled. The inter-corridor connections are highlighted as dashed lines. As observed, the network's topology is fully retained in this decomposition.

In Figure 3c one can see an excerpt of the multi-dimensional variational graph $G$ for corridors $C_{3}$ and $C_{1}$. Red and green phases are represented by the corresponding colors at the inter-corridor connections. The grey plane illustrates the inter-corridor connection $I_{2}$, and a single trajectory from a vehicle changing from one corridor to the other is shown as a dashed line for illustration purposes.

\subsection{Step 2: Integration of inter-corridor connections}

Once the multi-dimensional variational graph $G$ is defined, we apply nVT to solve the KWT problem. The original formulation, introduced in Section 2, cannot be applied as it does not account for discrete source terms. 
The implications of source terms for variational theory and the underlying concept of the Moskowitz function are shown in subsections 3.2.1 and 3.2.2. Mathematical formulations for upstream and downstream traffic state propagation across inter-corridor connections are presented in subsections 3.2.3 and 3.2.4, respectively.

\subsubsection{Discontinuous Moskowitz function}

In this section, we highlight the effects of source terms on the Moskowitz function on corridors by means of two examples, a 'free-flow case' and a 'congested case'.

Consider two uni-directional corridors $C_{i}$ and $C_{j}$ that intersect each other at the signalized inter-corridor connection $I_{i j}$ as displayed in Figure 4a. As there exist no bottlenecks on the corridors upstream of $I_{i j}$, we refer to this example as the 'free-flow case'. Let us denote the Moskowitz function at the position right downstream of $I_{i j}$ on each corridor as $N_{d}^{i}$ and $N_{d}^{j}$, respectively. The Moskowitz function for positions right upstream of $I_{i j}$ are $N_{u}^{i}$ and $N_{u}^{j}$, respectively. We further assume a high demand on both corridors, such that the inter-corridor connection is saturated. Moreover, we consider a case where all vehicles from $C_{j}$ merge onto $C_{i}$, i.e. the turning ratio $\alpha_{j i}=1$, and all vehicles on corridor $i$ continue straight at the inter-corridor connection, i.e. $\alpha_{i j}=0$. Figure 5b depicts possible time series of $N_{d}^{i}, N_{u}^{i}, N_{d}^{j}$ and $N_{u}^{j}$.

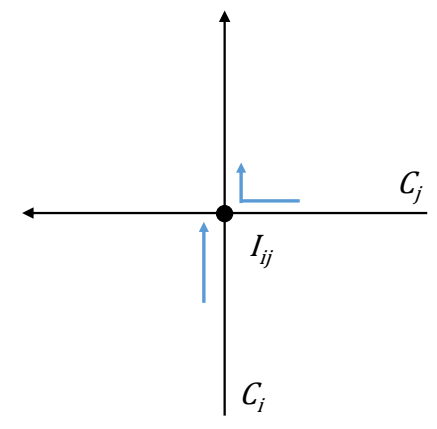

a) Two corridors $C_{i}$ and $C_{j}$ and a simple inter-corridor connection.

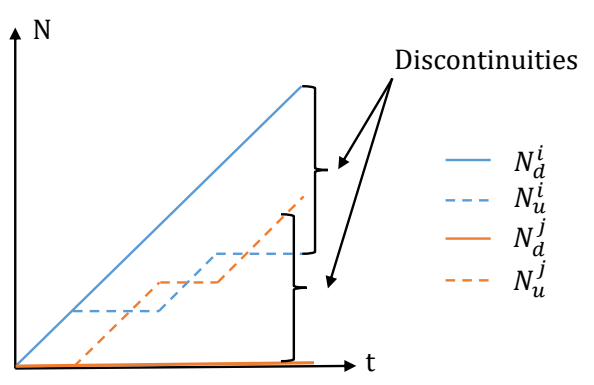

b) Moskowitz function $N$ for the upstream and downstream demands.

Figure 4: Free-flow case: network and Moskowitz function.

The Moskowitz functions $N_{u}^{i}$ and $N_{u}^{j}$ at the upstream positions show discharge flows during green phases, and zero flows during red phases. The downstream Moskowitz function for $C_{i}$ is the sum of both upstream functions, i.e. $N_{d}^{i}=N_{u}^{i}+N_{u}^{j}$, assuming that the travel time between $u$ and $d$ is negligible. The Moskowitz function downstream of the inter-corridor connection on corridor $C_{j}$ equals to zero, i.e. $N_{d}^{j}=0$, since no vehicles enter from $C_{i}$ and all flow on $C_{j}$ turns at the inter-corridor connection $I_{i j}$.

Recall that the Moskowitz function is in general continuous and respects the flow conservation principle (see Section 2). Since this assumption also applies to the original VT, it requires a continuous surface across space $x$ and time $t$. However, in our example, a discontinuity of the function occurs at the inter-corridor connection $I_{i j}$, as $N$ on the same corridor jumps drastically from upstream to downstream of $I_{i j}$ (see Figure 4b). In other words, no flow conservation applies considering each corridor separately although flow is indeed conserved at the network level, i.e. when flows are aggregated across the two corridors. Note that the discontinuous change in $N$ is positive when a net inflow occurs (e.g. at corridor $C_{i}$ ), and negative when a net outflow occurs (e.g. at corridor $C_{j}$ ). Additionally, the absolute value of the discontinuity increases with time depending on the volumes of the transfer flows.

The second example is the 'congested case' which is depicted in Figure 5. We examine a simple network with active bottlenecks somewhere downstream of the inter-corridor connection $I_{i j}$, from which congestion propagates upstream, as schematically illustrated in Figure 5a. The bottlenecks on each corridor are displayed by the black points, while the bold black arrow represents the back-propagation of congestion. Figure 5b shows possible time-series of the Moskowitz functions on both corridors, upstream and downstream of $I_{i j}$, i.e. $N_{d}^{i}, N_{u}^{i}, N_{d}^{j}$ and $N_{u}^{j}$.

In the figure, the occurrence of the spillback appears as a reduction in $N_{d}^{i}$ growing from $t=t_{c}$ onward which we denote as $\Delta N_{d}^{i}$. To ensure the correct propagation of spillbacks across inter-corridor connections, we have 


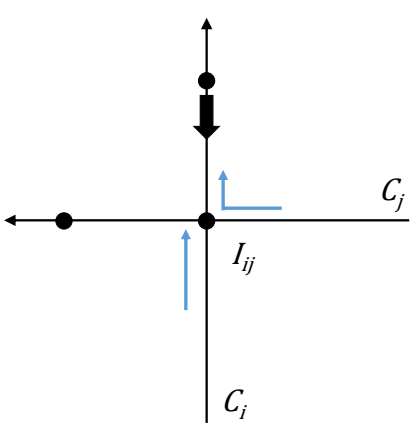

a) Two corridors $C_{i}$ and $C_{j}$, including two bottlenecks, and a simple inter-corridor connection.

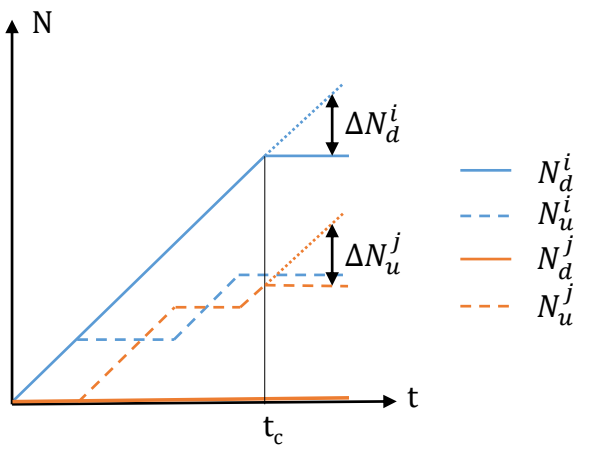

b) Moskowitz function $N$ for the upstream and downstream demands.

Figure 5: Congested case: network and Moskowitz function.

to account for the discontinuity at $I_{i j}$. Moreover, we have to define how to apportion $\Delta N_{d}^{i}$ into the $N_{u}$ of each corridor. As discussed in the previous section, $N_{d}^{i}$ is the sum of $N_{u}^{i}$ and $N_{u}^{j}$. In order to divide $\Delta N_{d}^{i}$ into the summands, we have to consider its time-dependency. Flows from the connected corridors can never occur simultaneously for the case of a signalized intersection without partially conflicting flows. This implies that congestion only propagates to the currently discharging corridor. Thus, only the Moskowitz function of this corridor is adapted. In our example, congestion is propagated from $N_{d}^{i}$ to $N_{u}^{j}$, i.e. $N_{u}^{j}$ is reduced by $\Delta N_{u}^{j}=\Delta N_{d}^{i}$. Therefore, $\Delta N_{d}^{i}$ is transferred to $N_{u}^{j}$, while $N_{u}^{i}$ stays constant until corridor $C_{i}$ gets the green light at the inter-corridor connection.

These examples for the 'free-flow case' and the 'congested case' highlight the existence of a discontinuity of the Moskowitz function at inter-corridor connections. Moreover, they show that the propagation of spillbacks necessitates the knowledge of the current signal phase. We have to extend the concept of VT to a discontinuous Moskowitz function to be able to solve the KWT represented by the variational graph $G$. In the following, we propose a framework to cope with this discontinuity for both the downstream and upstream traffic state propagation. This ensures a complete model including network-wide effects of spillbacks. For the sake of clarity, we avoid using the indices $i, j$ as much as possible for the explanations below, and only use them when strictly necessary. We will show that our methodology inherits the numerical exactness from the original VT for triangular FDs.

\subsubsection{Multi-dimensional variational graph at inter-corridor connections}

To further examine the effects of the discontinuous Moskowitz function, the analysis of corresponding excerpts of the variational graph $G$ is convenient. We define the location of an inter-corridor connection $I_{i j}$ in $G$ as $x_{\Phi}$. At this position, the turning ratio $\alpha_{i j}$ determines which portion of the flow is transferred from one corridor to another. Therefore, $x=x_{\Phi}+\Delta x$ is the first location in $G$ for a specific corridor, where $N$ is influenced by transfer flows. In other words, the discontinuity is located between $x_{\Phi}$ and $x_{\Phi}+\Delta x$.

We recall that eq.(4) is valid as long as the Moskowitz function is continuous. It is then valid for all $(x, t)$, where $x$ is not in the vicinity of the inter-corridor connection, i.e. $x \neq x_{\Phi}$ and $x \neq x_{\Phi}+\Delta x$. Between those positions, a discontinuity exists and the original formulation has to be modified as per the following two criteria. First, recall that the graphical interpretation of eq. (4) consists of three from-nodes and the corresponding edges. In order to account for transfer flows in the case of multiple corridors, eq.(4) should also reflect the traffic states from the adjacent corridor and the turning ratios. Second, eq.(4) cannot be evaluated at $x_{\Phi}$ and $x_{\Phi}+\Delta x$ because the edges in $G$ related to the terms of the minimum operation cross the discontinuity which violates the underlying assumptions of VT. This is shown in Figure 6. Thus, eq.(4) must be modified for $x=x_{\Phi}$ and $x=x_{\Phi}+\Delta x$.

Figure 6 depicts two excerpts of the multi-dimensional variational graph $G$ for corridors $C_{i}$ and $C_{j}$ around the inter-corridor connection located at $x_{\Phi}$. Edges which cross the discontinuity are shown as dashed lines. The terms in the minimum operation of eq.(4) corresponding to these dashed edges need to be modified in order to account for the existence of the discontinuity. The black color represents edges which are evaluated for corridor $C_{i}$, and the grey those being evaluated for corridor $C_{j}$. We label the points which have to be 


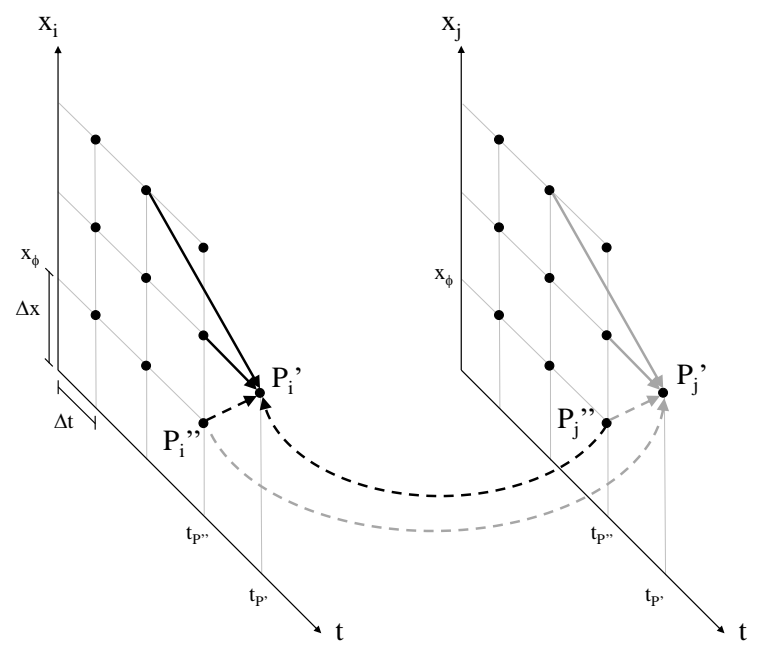

a) Downstream propagation of traffic states.

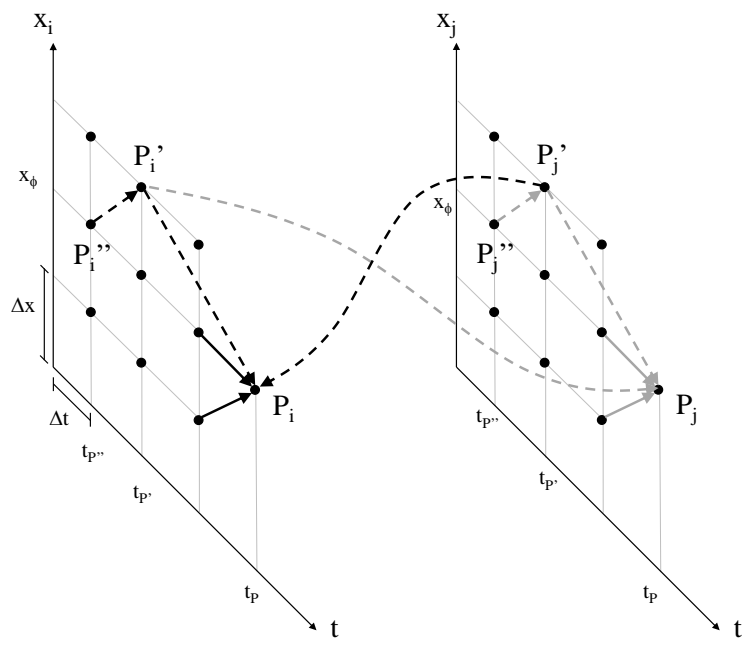

b) Upstream propagation of traffic states.

Figure 6: Excerpts of the multi-dimensional variational graph $G$ at the inter-corridor connection located at $x_{\Phi}$.

treated differently from the original formulation. These points are $P^{\prime \prime}$ at $\left(x_{\Phi}, t_{P^{\prime \prime}}\right), P^{\prime}$ at $\left(x_{\Phi}+\Delta x, t_{P^{\prime}}\right)$, and $P$ at $\left(x_{\Phi}, t_{P}\right)$. Note that $t_{P^{\prime \prime}}=t_{P}-(1+\theta) \Delta t, t_{P^{\prime}}=t_{P}-\theta \Delta t$, and $t_{P^{\prime \prime}}=t_{P^{\prime}}-\Delta t$. The explanations in Section 3.2.3 and 3.2.4 refer to these points.

Figure 6a depicts the propagation of traffic states from upstream nodes (i.e. free-flow traffic states). This propagation is affected by the discontinuity when the corresponding edges start at $x_{\Phi}$ and end at $x_{\Phi}+\Delta x$. Thus, the first term in eq.(4) has to be modified when calculating $N(x, t)$ for $x=x_{\Phi}+\Delta x$. Figure $6 \mathrm{~b}$ shows the propagation of traffic states from downstream nodes (i.e. congested traffic states). It is affected by the discontinuity when the corresponding edges start at $x_{\Phi}+\Delta x$ and end at $x_{\Phi}$. Consequently, the second term in eq. (4) has to be modified when calculating $N(x, t)$ for $x=x_{\Phi}$. The following subsections describe this extension of eq. (4) for the propagation of traffic states from upstream and downstream nodes in the vicinity of the inter-corridor connection, i.e. at $x=\left\{x_{\Phi}, x_{\Phi}+\Delta x\right\}$.

\subsubsection{Propagation of traffic states from upstream nodes at $x=x_{\Phi}+\Delta x$}

This section explains how to include source terms when traffic states propagate from upstream nodes across the discontinuity of the Moskowitz function. In this case, the first term in the minimum operator in eq.(4) becomes decisive, as it refers to the free-flow states traveling from upstream. We modify this term at $x=x_{\Phi}+\Delta x$ to account for the upstream demand, and incorporate inflows and outflows based on turning ratios at inter-corridor connections.

Recall that each term in eq.(4) consists of a known $N$ and the costs $c$ along a path. In order to modify the equation, we adapt these two elements for its first term.

First, we focus on the known $N$-value at the upstream node, which equals to $N_{P^{\prime \prime}}$ following the designations in Figure 6a. We assume that $N$ is known for all $t<t_{P^{\prime}}$. This is feasible because we can solve for $N$ moving from left to right in the variational graph. The turning ratio $\alpha_{i j}$ specifies the transfer flow from $x_{i, \Phi}$ to $x_{j, \Phi}+\Delta x$. Similarly, the turning ratio $\alpha_{j i}$ specifies the transfer flow from $x_{j, \Phi}$ to $x_{i, \Phi}+\Delta x$. The demand at $P_{i}^{\prime}$ can be calculated as the sum of the flow which stays on the corridor $C_{i}$, i.e. $N_{P^{\prime \prime}}^{i} \cdot\left(1-\alpha_{i j}\right)$, and the inflow from corridor $C_{j}$, i.e. $N_{P^{\prime \prime}}^{j} \cdot \alpha_{j i}$. This is depicted by the black dashed lines in Figure 6 a for $C_{i}$.

Second, we examine the costs $c$ associated to the path from $N_{P^{\prime \prime}}$ to $N_{P^{\prime}}$. Note that $c=0$ for the propagation of traffic states from upstream nodes, as such states correspond to free-flow conditions. This fact does not change by considering inflows, and therefore the costs for these edges remain equal to zero.

Eventually, we determine the term $N_{P^{\prime}}^{i}$ by evaluating the following equation: 


$$
\begin{aligned}
N_{P^{\prime}}^{i}\left(x_{\Phi}+\Delta x, t_{P^{\prime}}\right)=\min & \left(N_{P^{\prime \prime}}^{i}\left(x_{\Phi}, t_{P^{\prime \prime}}\right) \cdot\left(1-\alpha_{i j}\right)+N_{P^{\prime \prime}}^{j}\left(x_{\Phi}, t_{P^{\prime \prime}}\right) \cdot \alpha_{j i},\right. \\
& N^{i}\left(x_{\Phi}+2 \Delta x, t-\theta \Delta t\right)+\Delta x \kappa, \\
& \left.N^{i}\left(x_{\Phi}+\Delta x, t-\Delta t\right)+\beta\right) .
\end{aligned}
$$

The term $N_{P^{\prime}}^{j}$ can be determined analogously. For both corridors, it can be seen that this equation only requires to consider $N_{P^{\prime \prime}}$ at the position of the inter-corridor connection $x_{\Phi}$. All other $N$ are known from the original VT formulation. Note that signal phases do not have to be considered explicitly as they are already reflected in the values of $N_{P^{\prime \prime}}^{i}$ and $N_{P^{\prime \prime}}^{j}$, i.e. they are implicitly taken into account.

\subsubsection{Propagation of traffic states from downstream nodes at $x=x_{\Phi}$}

In order to complete our modeling framework, we have to ensure the correct propagation of traffic states from downstream nodes across the inter-corridor connections where discontinuities of the Moskowitz function appear. Recall that only congested traffic states can be propagated from downstream. Thus, we modify the second term in eq.(4) for $x=x_{\Phi}$ which is decisive when congestion occurs. In other words, in this section we explain how to model spillbacks. Without the loss of generality, the formulation in the following refers to corridor $C_{i}$ only. The formulation applies analogously for evaluating the traffic conditions on corridor $C_{j}$. For the sake of clarity, we avoid using the coordinates of the points $P^{\prime \prime}, P^{\prime}$, and $P$ in the following formulations except for the eq.(11). They are described in subsection 3.2.3 and illustrated in Figure 6.

Again, the second term of the minimum operation consists of two elements. The first element is the known Moskowitz function value $N_{P^{\prime}}$ at the downstream node $P^{\prime}\left(x_{\Phi}+\Delta x, t-\theta t\right)$. The second one refers to the $\operatorname{costs} c$, i.e. the number of vehicles which can pass a moving observer travelling from the downstream node $P^{\prime}$ to the point $P\left(x_{\Phi}, t\right)$.

We first focus on the value $N_{P^{\prime}}$. Notice that any changes in $N_{P^{\prime}}$, i.e. $\Delta N_{P^{\prime}}$ (see Figure $5 \mathrm{~b}$ ) should first be apportioned to each of the corridors upstream of $I_{i j}$ according to the signal phases and turning ratios. In the absence of congestion the portion of $N_{P^{\prime}}$ which corresponds to the Moskowitz surface related to $N_{P}$ would simply be $N_{P^{\prime \prime}} \cdot\left(1-\alpha_{i j}\right)$ as per the first term in eq.(5). The effect of congestion can be described as a reduction of $N$, denoted as $\Delta N$ in Figure $5 \mathrm{~b}$. Since we know $N_{P^{\prime}}$ and $N_{P^{\prime \prime}}$, we can calculate $\Delta N$. Note that corresponding spillbacks can originate on both corridors $C_{i}$ and $C_{j}$. Thus, we have to consider two different scenarios for each corridor. For corridor $C_{i}$ we can write:

$$
\begin{aligned}
& \Delta N_{P^{\prime}}^{i i}=\left(N_{P^{\prime \prime}}^{i} \cdot\left(1-\alpha_{i j}\right)+N_{P^{\prime \prime}}^{j} \cdot \alpha_{j i}\right)-N_{P^{\prime}}^{i}, \\
& \Delta N_{P^{\prime}}^{i j}=\left(N_{P^{\prime \prime}}^{i} \cdot \alpha_{i j}+N_{P^{\prime \prime}}^{j} \cdot\left(1-\alpha_{j i}\right)\right)-N_{P^{\prime}}^{j} .
\end{aligned}
$$

Recall that VT is built upon the concept of the moving observer. Keeping this notion in mind, one can interpret $\Delta N_{P^{\prime}}$ as the difference in the number of vehicles a moving observer traveling from $N_{P^{\prime \prime}}$ to $N_{P^{\prime}}$ passes due to congestion compared to free-flow conditions. The indices $i$ and $j$ correspond to the corridors where flows originate and propagate to, respectively. For example, $\Delta N_{P^{\prime}}^{i j}$ relates to vehicles in congestion coming from corridor $C_{i}$ with destination in corridor $C_{j} . \Delta N_{P^{\prime}}^{i i}$ relates to vehicles in congestion coming from $C_{i}$ with destination in corridor $C_{i}$. Note that the portion of $N_{P^{\prime}}$ related to the upstream Moskowitz functions on each corridor is determined based on the respective turning ratios $\alpha$, as shown for the free-flow case in eq.(5). This has to be considered when the change in the vehicle number $\Delta N_{P^{\prime}}$ is apportioned. We do so by dividing $\Delta N_{P^{\prime}}$ by the turning ratio associated to each corridor. As an example assume a turning ratio of $\alpha_{i j}=50 \%$ and a $\Delta N_{P^{\prime}}^{i j}=5$ vehicles. That would mean that 5 vehicles coming from $P_{i}^{\prime \prime} \operatorname{did}$ not reach $P_{j}^{\prime}$ due to congestion. Moreover, this $\Delta N_{P^{\prime}}^{i j}=5$ would mean that 10 vehicles departed $P_{i}^{\prime \prime}$ as only every second vehicle wanted to travel to $P_{j}^{\prime}$. To find then the portion of $N_{P^{\prime}}$ corresponding to the Moskowitz function related to $N_{P}$, denoted as $\widehat{N}_{P^{\prime}}$ in eq.(7), we subtract from $N_{P^{\prime \prime}}$ the total number of vehicles leaving $P^{\prime \prime}$ being blocked by the congestion at $P^{\prime}$. This lets us formulate the effects of congestion at $P^{\prime}$ related to the Moskowitz function at $x_{\Phi}$. 


$$
\begin{aligned}
& \widehat{N}_{P^{\prime}}^{i i}=N_{P^{\prime \prime}}^{i}-\Delta N_{P^{\prime}}^{i i} \cdot \frac{1}{1-\alpha_{i j}}, \\
& \widehat{N}_{P^{\prime}}^{i j}=N_{P^{\prime \prime}}^{i}-\Delta N_{P^{\prime}}^{i j} \cdot \frac{1}{\alpha_{i j}} .
\end{aligned}
$$

To find the upper bound for $N_{P}$ due to congestion, we further have to consider the costs along the path from $P^{\prime}$ to $P$ which corresponds to the second element of the term related to congestion in eq.(4). Again, we consider the case of congestion propagation only on corridor $C_{i}$. Note that the costs are $\Delta x \kappa$ in the original formulation. In our case, these costs only apply when the signal phase does not change during the interval $[t-(1+\theta) \Delta t, t]$, i.e. in the time interval between $N_{P^{\prime \prime}}$ and $N_{P}$. However, when a signal phase change occurs during that interval, flows from both corridors might occur at different times within the interval. Thus, the costs $c$ include vehicles originating from both corridors. We need to apportion $c$ according to the vehicles' origin for a correct spillback propagation. In other words, we need to ensure that costs only reflect the vehicles related to the corridor where congestion is propagating to. Otherwise, we would overestimate the costs of the edge, and consequently the second term in eq.(4). Ultimately, it could result in an overestimation of $N_{P}$ and thus in an erroneous traffic state propagation from downstream. Assuming reasonable backward wave speeds and that there is only one signal phase change during the considered time interval, there are only two possibilities for such a phase change to occur:

- Change from red to green: In this case, a green phase is active at $t=t_{P}$. The correct maximum number of vehicles from $C_{i}$ that a moving observer travelling from $P_{i}^{\prime}$ to $P_{i}$ and from $P_{j}^{\prime}$ to $P_{i}$ would count has thus to be reduced by the vehicles coming from $C_{j}$. Let the variable $r$ denote the duration of the red phase for $C_{i}$ starting at $t=t_{P^{\prime \prime}}=t_{P}-(1+\theta) \Delta t$. This is equivalent to the green time for $C_{j}$ for that same period. Then, the maximum number of vehicles discharging from $C_{j}$ and going to $C_{i}$ is $\Delta x \kappa \cdot \frac{r}{(1+\theta) \Delta t} \cdot \alpha_{j i}$. Consequently, the maximum number that can discharge from $C_{i}$ and stay on that corridor is $\Delta x \kappa-\Delta x \kappa \cdot \frac{r}{(1+\theta) \Delta t} \cdot \alpha_{j i}$. As for $\Delta N_{P^{\prime}}$, we consider that a part of the total inflow on corridor $C_{i}$ stays on that corridor and a part turns into corridor $C_{j}$, so we divide by the appropriate turning ratios, i.e. $1-\alpha_{i j}$ for flow staying in $C_{i}$ and $\alpha_{i j}$ for flow from $C_{i}$ to $C_{j}$. The costs $c$ can then be formulated as:

$$
\begin{aligned}
c^{i i} & =\frac{1}{1-\alpha_{i j}} \cdot\left(\Delta x \kappa-\Delta x \kappa \cdot \frac{r}{(1+\theta) \Delta t} \cdot \alpha_{j i}\right), \\
c^{i j} & =\frac{1}{\alpha_{i j}} \cdot\left(\Delta x \kappa-\Delta x \kappa \cdot \frac{r}{(1+\theta) \Delta t} \cdot\left(1-\alpha_{j i}\right)\right) .
\end{aligned}
$$

- Change from green to red: In this case, a red phase is active at $t=t_{P}$. Let the variable $g$ denote the duration of the green phase starting at $t=t_{P^{\prime \prime}}=t_{P}-(1+\theta) \Delta t$. Then, the maximum number of vehicles discharging from $C_{i}$ and staying on this corridor is $\Delta x \kappa \cdot \frac{g}{(1+\theta) \Delta t} \cdot\left(1-\alpha_{i j}\right)$. Analogously to the previous case, an formulation for the costs $c$ can be found. However, for the case of an active red phase at $t=t_{P}$, the term related to capacity constraints in eq.(4) becomes decisive. Therefore, and for the sake of clarity, we do not explicitly state the corresponding mathematical formulation in this paper.

The sum of $\widehat{N}_{P^{\prime}}$ from eq.(7) and the costs $c$ from eq.(8) is an important step for the final formulation of the spillback-induced upper bound for $N_{P}$. This sum can be written as:

$$
\begin{gathered}
\widehat{N}_{P^{\prime}}^{i i}+c^{i i}=N_{P^{\prime \prime}}^{i}+\frac{1}{1-\alpha_{i j}} \cdot\left[\Delta x \kappa \cdot\left(1-\frac{r}{(1+\theta) \Delta t} \cdot \alpha_{j i}\right)-\Delta N_{P^{\prime}}^{i i}\right], \\
\widehat{N}_{P^{\prime}}^{i j}+c^{i j}=N_{P^{\prime \prime}}^{i}+\frac{1}{\alpha_{i j}} \cdot\left[\Delta x \kappa \cdot\left(1-\frac{r}{(1+\theta) \Delta t} \cdot\left(1-\alpha_{j i}\right)\right)-\Delta N_{P^{\prime}}^{i j}\right] .
\end{gathered}
$$

Note that the term in the squared brackets can become negative. This is for example the case when $\Delta N_{P^{\prime}}=\Delta x \kappa$, i.e. the space between $P^{\prime \prime}$ and $P^{\prime}$ is fully congested, and both $r$ and $\alpha$ are unequal to zero. Since the Moskowitz function is a monotonically increasing function, such values are impossible. We avoid 
them by applying a maximum function on the term in the squared brackets. Then, we can describe the influence of congestion from corridors $C_{i}$ and $C_{j}$ on the Moskowitz function $N_{P}^{i}$ as:

$$
\begin{gathered}
N_{P}^{i}=N_{P^{\prime \prime}}^{i}+\frac{1}{1-\alpha_{i j}} \cdot \max \left(0, \Delta x \kappa \cdot\left(1-\frac{r}{(1+\theta) \Delta t} \cdot \alpha_{j i}\right)-\Delta N_{P^{\prime}}^{i i}\right), \\
N_{P}^{i}=N_{P^{\prime \prime}}^{i}+\frac{1}{\alpha_{i j}} \cdot \max \left(0, \Delta x \kappa \cdot\left(1-\frac{r}{(1+\theta) \Delta t} \cdot\left(1-\alpha_{j i}\right)\right)-\Delta N_{P^{\prime}}^{i j}\right) .
\end{gathered}
$$

This equation formally corresponds to the intuition that we have to consider the different Moskowitz functions upstream and downstream of the discontinuity, as well as the current signal phases. Eq.(10a) becomes constraining when spillbacks come from corridor $C_{i}$, and eq.(10b) becomes constraining for spillbacks from corridor $C_{j}$. The general formulation for $N_{P}\left(x_{\Phi}, t\right)$ includes then eq.(10), as well as the free-flow and the capacity related terms.

$$
\begin{aligned}
N_{P}^{i}\left(x_{\Phi}, t\right)=\min & \left(N^{i}\left(x_{\phi}-\Delta x, t-\Delta t\right),\right. \\
& N_{P^{\prime \prime}}^{i}\left(x_{\phi}, t-(1+\theta) \Delta t\right)+\frac{1}{1-\alpha_{i j}} \cdot \max \left(0, \Delta x \kappa \cdot\left(1-\frac{r}{(1+\theta) \Delta t} \cdot \alpha_{j i}\right)-\Delta N_{P^{\prime}}^{i i}\right), \\
& N_{P^{\prime \prime}}^{i}\left(x_{\phi}, t-(1+\theta) \Delta t\right)+\frac{1}{\alpha_{i j}} \cdot \max \left(0, \Delta x \kappa \cdot\left(1-\frac{r}{(1+\theta) \Delta t} \cdot\left(1-\alpha_{j i}\right)\right)-\Delta N_{P^{\prime}}^{i j}\right), \\
& \left.N^{i}\left(x_{\phi}, t-\Delta t\right)+\beta\right) .
\end{aligned}
$$

\subsection{Implementation}

The derived equations enable us to numerically derive the KWT solution for a network with turning flows. The corresponding pseudo-algorithm is shown in algorithm 1. It describes the application of the proposed equations for a network $\mathcal{N}$. Based on this network, the set of corridors $\mathcal{C}$ is derived. It includes all turning ratios $A$, the control settings, and the inflow at origins. For a total simulation period $T$, one iterates through all $t$ for each corridor $C$ and evaluates eq.(4) - eq.(11).

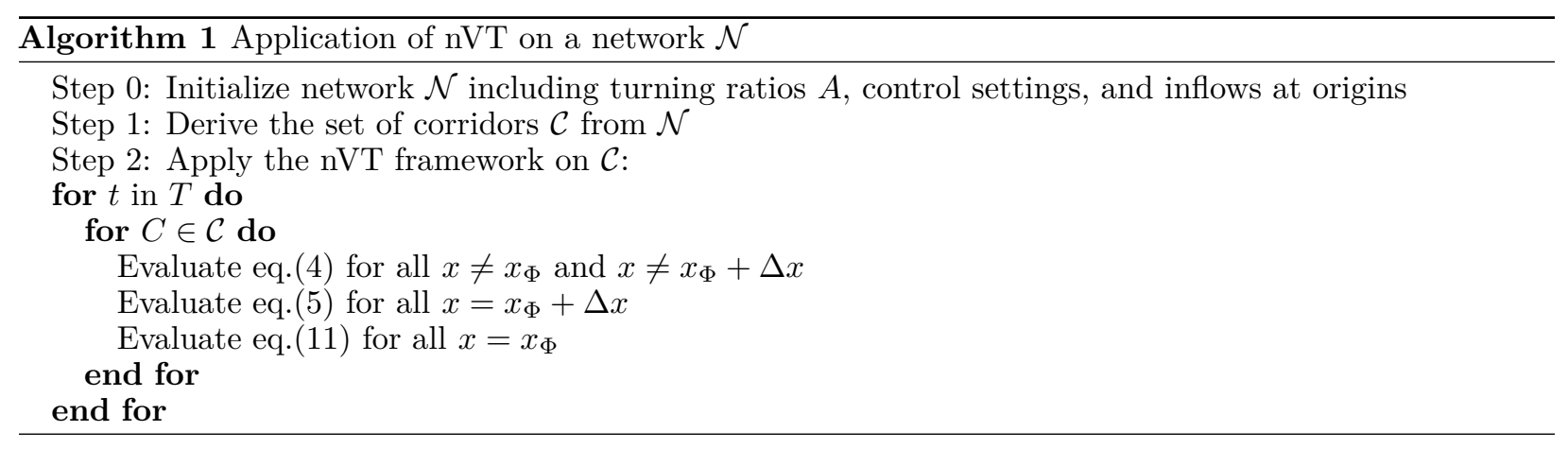

This nVT model allows to solve complex heterogeneous KWT problems related to networks with signalized control. Such problems involve source terms which are discrete in time and space. The required inputs are the triangular FD, network topology, control settings, and demand patterns including turning ratios. As VT allows the incorporation of any type of bottlenecks, $\mathrm{nVT}$ inherits this ability. The exact evaluation of $N(x, t)$ makes it superior to other methods like the CTM. The ability to handle complex heterogeneities at the link level is advantageous compared to the LTM. Similar to the original VT framework, the computational effort of our nVT only scales with time and space. This is beneficial compared to microscopic simulations which usually also scale with the number of vehicles and thus congestion occurrence. We will discuss these points in more detail in the next sections. 


\section{Evaluation of $\mathrm{nVT}$ and its accuracy}

In this section, we investigate the performance of our nVT on a small toy network, including the incorporation of source terms and the propagation of congestion across inter-corridor connections. Moreover, we analyze the role of the time-step size $\Delta t$ on the accuracy of the framework.

\subsection{Proof of concept}

As a proof of concept, we now test our nVT model for a 'free-flow scenario' and a 'congested scenario' on the networks depicted in Figure 7 . The network on the left consists of two corridors, $C_{1}$ and $C_{2}$, that intersect each other at the inter-corridor connection $I_{1}$. Recall that we defined inter-corridor connections as intersections with turning ratios $\alpha>0$. While the right-hand network is similar, two additional intersections exist, one on each corridor. These intersections act as active bottlenecks downstream of the inter-corridor connection $I_{2}$ and therefore lead to congestion. The corridors are labelled as $C_{3}$ and $C_{4}$, respectively. The designation of the two scenarios refers to the occurring traffic states downstream of the inter-corridor connection. We assume possible turning movements for both inter-corridor connections $I_{1}$ and $I_{2}$, as illustrated by the blue arrows in the figure. We set a cycle length of $90 \mathrm{~s}$, and a red and green phase of $45 \mathrm{~s}$ for all approaches at all intersections. All offsets are set to zero. We assume a triangular FD with jam density $\kappa=150$ veh $/ \mathrm{km}$, a free-flow speed $u=10 \mathrm{~m} / \mathrm{s}$ and a backward wave speed $w=5 \mathrm{~m} / \mathrm{s}$. The inflow demand is set to a volume-to-capacity ratio of $V O C=1.0$, which refers to the intersection capacity, for both scenarios.

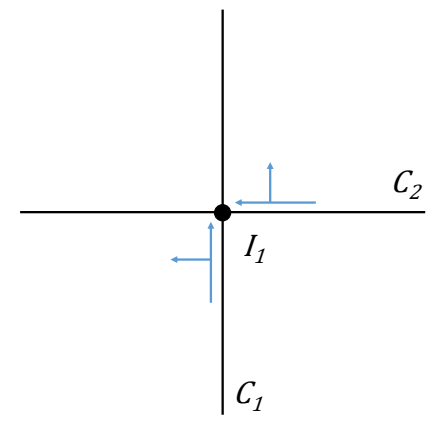

a) Free-flow scenario.

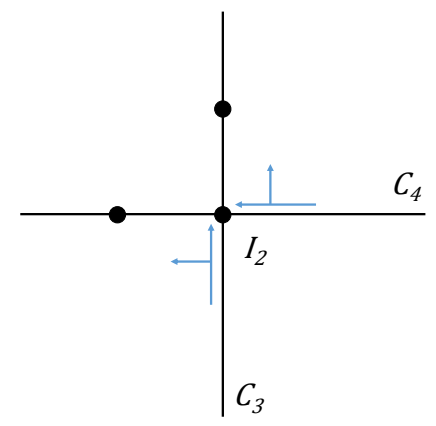

b) Congested scenario.

Figure 7: Test networks.

In the following, we first evaluate the traffic dynamics for the network displayed in Figure 7a. We discuss our implementation of source flows for the case where no congestion is propagated from downstream of $I_{1}$ (Section 4.1.1). We then analyze the results for the congested case (see Figure 7b), where the bottlenecks downstream of $I_{1}$ are active (Section 4.1.2). This will confirm that our proposed nVT model is able to capture traffic dynamics in its full range including spillbacks, which occur due to the active bottlenecks. Additionally, we verify our results by reproducing the traffic conditions on the same networks (given its topology and control settings) with the microscopic simulator SUMO (Lopez et al., 2018) and an implementation of Newell's car-following model. This allows to show that the predicted traffic states using nVT are in agreement with the KWT solution for the test networks.

\subsubsection{Inclusion of source terms}

The evaluation of nVT for the first network enables us to isolate the effects of source terms for the case where no downstream congestion affects the traffic flow close to the inter-corridor connection. This allows us to study the effects of different turning ratios. We assume turning ratios of $\alpha_{1,2}=0.25$ and $\alpha_{2,1}=0.5$. The total simulated time is $T=500 \mathrm{~s}$, and the time-step length which determines the size of the multi-dimensional variational graph $G$ is $\Delta t=0.1 \mathrm{~s}$. Recall that this specifies $\Delta x$ as well.

Figure 8 shows the results for both corridors as contour plots. The x-axis displays time in seconds, and the $\mathrm{y}$-axis space in meters. The color bar represents the density ranging from bright yellow for low densities, to dark blue for high ones. These densities were obtained with the proposed nVT model. We also show the trajectories extracted from SUMO as black and white curves, on top of the contour plots. The black curves depict trajectories originating on corridor $C_{1}$, while the white ones correspond to those originating on $C_{2}$. The effects of signal phases at the intersection at $\mathrm{x}=250 \mathrm{~m}$ are clearly identified. The dark blue areas 
correspond to the jam density and thus represent the evolution of queues upstream of the inter-corridor connection on both corridors. The discharge flows colored in green can also be clearly observed in the figure. More important are the evident transfer flows between both corridors. This is illustrated by two facts. First, densities $k>0$ exist downstream of $I_{1}$ also during red phases. Second, the discharge flows are split at $I_{1}$, as depicted by the different densities upstream and downstream of $I_{1}$ for each corridor even during the green phase. This is highlighted by the change in colors. The trajectories from SUMO represent a perfect match to the predicted traffic states by our nVT model. They show that our framework successfully incorporates source terms for the case when congestion does not propagate to the inter-corridor connection.

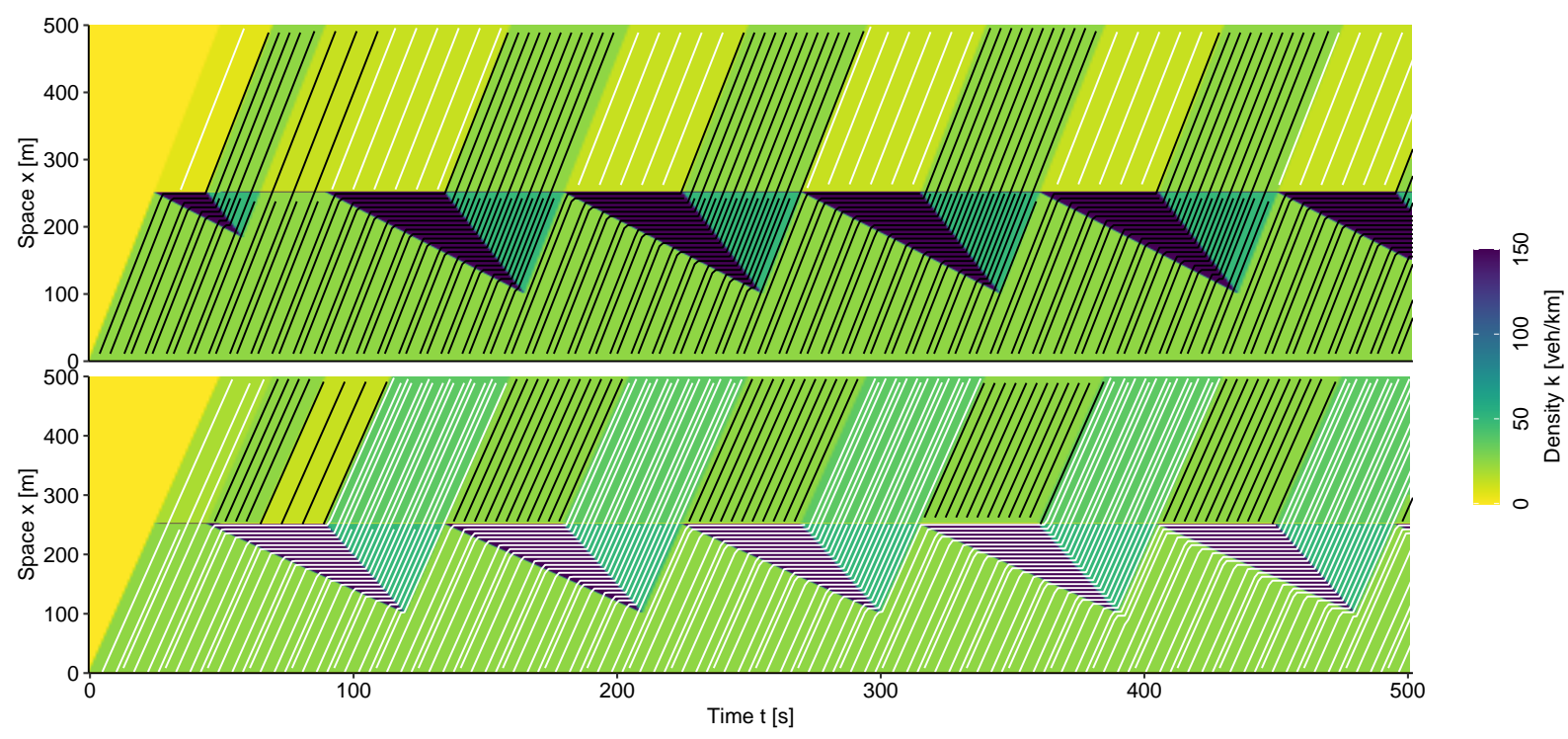

Figure 8: Free-flow scenario: Time-space diagrams for corridor $C_{1}$ (top) and corridor $C_{2}$ (bottom) with a traffic signal at $I_{1}(x=250 \mathrm{~m})$. The colors represent the density which was obtained with the nVT model. The curves indicate trajectories obtained with the microscopic traffic simulator, and their color indicates the origin (i.e black trajectories originate in $C_{1}$ and white ones originate in $C_{2}$ ).

\subsubsection{Propagation of spillbacks}

In the second step, we discuss a more general case where congestion appears downstream of the inter-corridor connection. Our proposed framework should propagate spillbacks along and across corridors. That is, the back of the queue has to spillover to at least one of the corridors, depending on the signal phase.

Figure 9 shows the simulation results. The axis and colors of the figure are the same as those of Figure 8 . The black curves depict trajectories originating on corridor $C_{3}$, while the white ones correspond to those originating on $C_{4}$. The graph clearly shows the effects of the additional signals at $\mathrm{x}=400 \mathrm{~m}$ which behave as active bottlenecks. The congestion starting on corridor $C_{3}$ propagates to $I_{2}$, where it affects both corridors upstream of $I_{2}$. This can be seen by the queues which grow on both corridors with each cycle. Again, the traffic states predicted by our nVT model fit the trajectories recorded from SUMO, illustrating that our framework is able to successfully propagate congestion across inter-corridor connections.

\subsection{Numerical error}

In order to solve a given KWT problem with the nVT model, we have to define a multi-dimensional variational graph $G$. The choice of the time-step size $\Delta t$ in $G$ is crucial for the computational cost. The original VT has the advantage of being exact in determining the Moskowitz function, independently of the grid size. The only existing error is the so-called 'sampling error' (Daganzo and Menendez, 2005) that originates from an inaccurate sampling of data along the boundary.

The incorporation of discrete source terms at inter-corridor connections does not alter the fact that the framework is based on VT. It also inherits the exact numerical calculation of the Moskowitz function, as well 


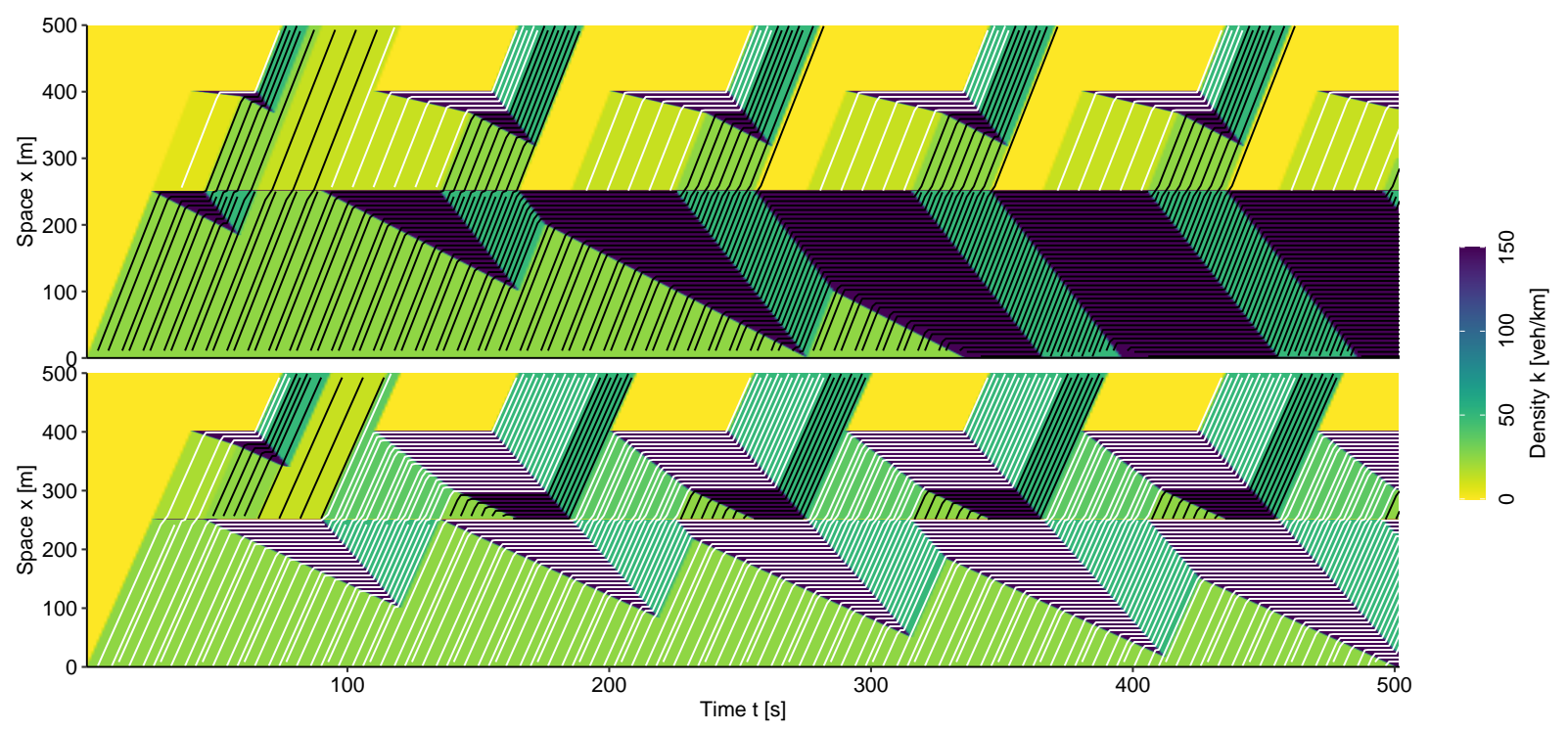

Figure 9: Congested scenario: Time-space diagrams for corridor $C_{3}$ (top) and corridor $C_{4}$ (bottom) with a traffic signal at $I_{2}(x=250 \mathrm{~m})$. The colors represent the density which was obtained with the nVT model. The curves indicate trajectories obtained with the microscopic traffic simulator, and their color indicates the origin (i.e black trajectories originate in $C_{3}$ and white ones originate in $C_{4}$ ).

as any potential sampling errors associated to VT. Our framework is able to consider inflows and outflows as long as the corresponding terms are mapped in the variational graph. This is not different to existing VT solutions, where bottlenecks are only considered when they are incorporated in $G$. Thus, nothing changes compared to the current VT techniques with regard to numerical errors. In order to illustrate this, we evaluate the congested scenario from the previous subsection for the time-steps $\Delta t=\{0.1,1,5\} s$. Note that the same analysis could be conducted for any other scenario. We plot $N$ at $I_{2}$ across $t$ for both corridors.

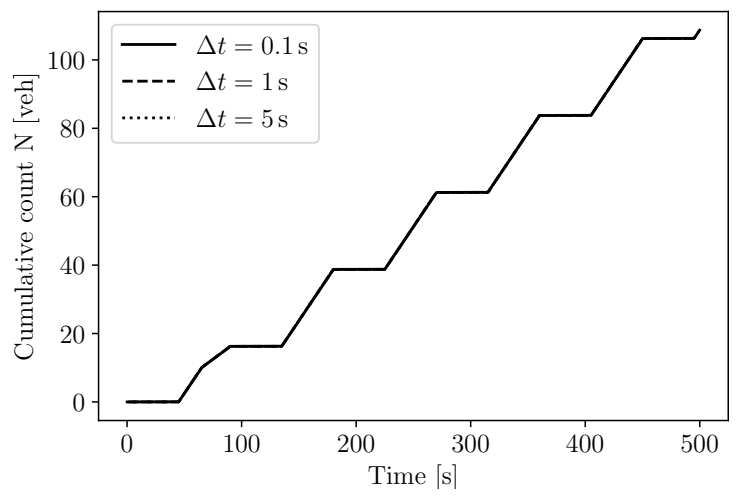

a) Moskowitz functions at $I_{2}$ on corridor $C_{3}$.

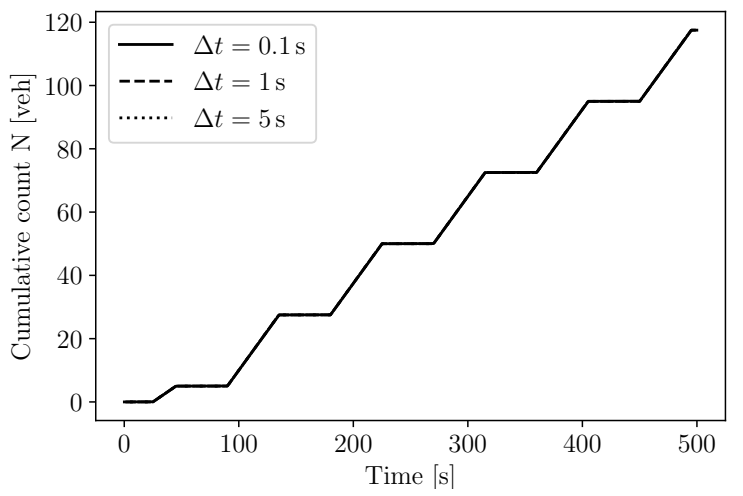

b) Moskowitz functions at $I_{2}$ on corridor $C_{4}$.

Figure 10: Impact of the time-step $\Delta t$ on the results.

The y-axis displays the Moskowitz function $N$, the x-axis shows the time $t$ in seconds. We plot the curves resulting from the different times-steps as solid, dashed and dotted lines. However, comparing the numerical values of all Moskowitz functions $N_{\Delta t=0.1}, N_{\Delta t=1}$, and $N_{\Delta t=5}$ reveals that there is no difference between any of them. The figure illustrates this by showing the exact same shape with a perfect overlap of all curves. In other words, the Moskowitz function $N(x, t)$ is found independently of the time-step $\Delta t$, as long as all bottlenecks and source terms are mapped in $G$. This confirms that our framework does not add any additional 
error to the original VT framework and thus is exact in determining $N$ given a triangular FD and no sampling error at the boundary.

\section{$5 \quad$ Application of $\mathrm{nVT}$ to a realistic network}

The previous section shows a very good fit of the trajectories from SUMO and the traffic states resulting from our proposed nVT model. This section further applies our method on a larger network and compares the predicted traffic states to the ones from a corresponding SUMO scenario. For our case study, we create a network based on the well-known Sioux Falls example ${ }^{1}$. The solution of the KWT is the Moskowitz function $N(x, t)$. It allows us to derive all sorts of indicators, such as average flows, densities, and speeds, but also travel times and delays. Thus, we present our results as Moskowitz functions in this case study. We compare the results derived from our VT framework to those obtained with SUMO. First, we measure the average flows per cycle according to both methods. This is a suitable aggregation for minimizing the stochastic aspects of SUMO. Second, we show the Moskowitz functions for three locations in the network for the purpose of illustration. Third, we analyze the computational effort of both methods, as the associated cost for macroscopic models is usually low and thus advantageous for numerous applications.

\subsection{Case study design}

The nature of our framework and of the microscopic simulation are fundamentally different. The former is macroscopic, and traffic can be deterministically assigned based on origin flows and turning ratios. Moreover, intersections are modelled as points in space. The latter includes a car-following model, as well as routing aspects next to origin flow and turning ratio definitions. In particular, SUMO allows specifying turning ratios at intersections which are then considered in an internal routing algorithm for each vehicle. However, deviations from the set turning ratios still occur. Also, intersections include radii with certain lengths, and are thus not modelled as points in space. Having these differences between the two models in mind, we apply several simplifications, described in the following, to be able to conduct a reasonable comparison.

Figure 11 presents the network for which the case study is performed. For the sake of simplicity, we only consider intersections with 4 or fewer legs, and thus reduce the original Sioux Falls network slightly. In total, the network consists of 43 links and 23 intersections. Each link is unidirectional and has only one lane. Note that our model is not limited to unidirectional networks. However, the implementation of bi-directional links increases the complexity of the case study, and therefore the influence of stochastic aspects of SUMO, e.g. turning ratios, would become more significant. Hence, we avoid bi-directional links to increase the comparability of the results from nVT and SUMO. All intersections are signalized with a cycle time of $90 \mathrm{~s}$, and a green and red phase of $45 \mathrm{~s}$. All offsets are set to zero. The direction of travel is indicated by the arrows in the figure.

In order to apply our nVT model, origin nodes and inflows, as well as turning ratios at inter-corridor connections are required. We define the origin nodes, highlighted with arrows in Figure 11. We evaluate the case study for five different demand scenarios with volume-to-capacity ratios of $V O C \in\{0.2,0.4,0.6,0.8,1.0\}$ where the capacity again refers to that of intersections. Additionally, we define turning ratios for five intersections, i.e. five inter-corridor connections. This number is chosen to further reduce the complexity of the problem as it limits the impact of vehicle routing in SUMO; thereby increasing the comparability of both methods. Turning ratios are set to $\alpha=0.5$ for all five inter-corridor connections. The inter-corridor connections are highlighted as dashed squares in Figure 11. Note that by defining origin nodes and turning ratios, no destination nodes need to be set explicitly. The simulation period is one hour. We choose a time-step of $\Delta t=0.1 \mathrm{~s}$, and an FD with the same parameters as described in the previous section.

Furthermore, as described in step 1 in section 3.1, we decompose the network into corridors based on the horizontal and vertical orientation of links. In total we define 12 corridors. We describe them by noting the included intersections in order of the travel direction in Table 1. Recall that the method of decomposition does not affect the final result of $\mathrm{nVT}$.

\footnotetext{
${ }^{1}$ derived from: https://github.com/bstabler/TransportationNetworks
} 


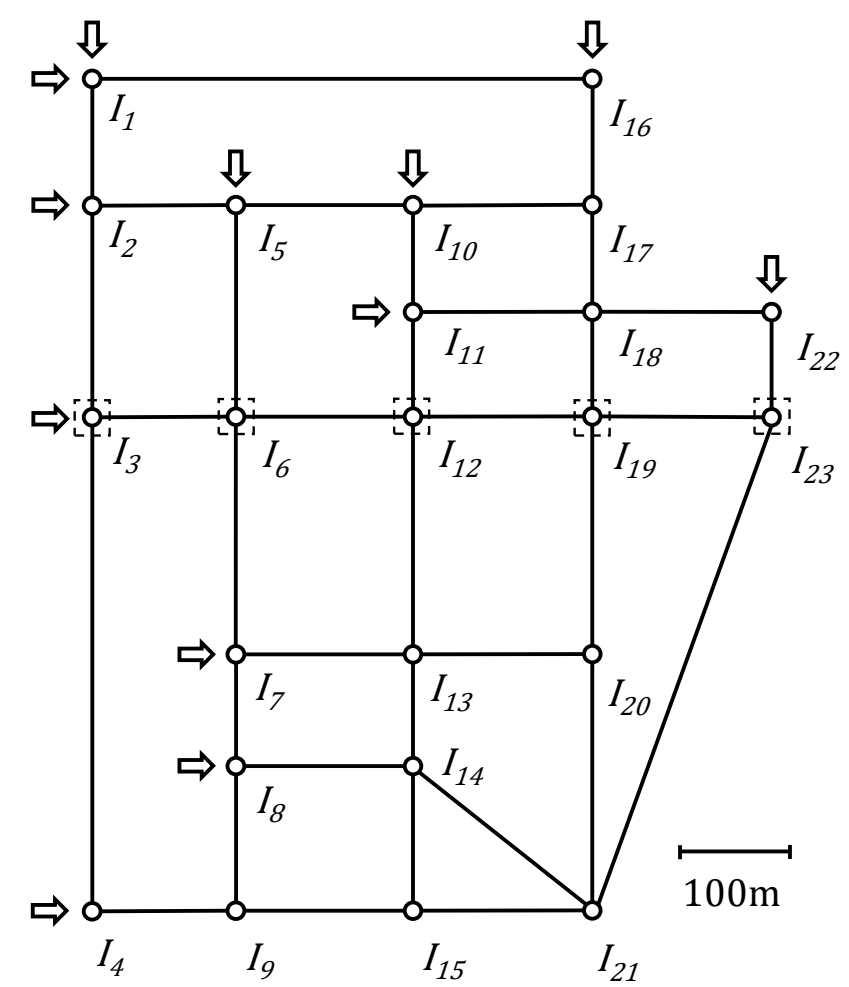

Figure 11: Sioux Falls network for the case study.

Table 1: Network decomposition.

\begin{tabular}{lr}
\hline Corridor $C \in \mathcal{C}$ & Intersections $I \in \mathcal{I}$ \\
\hline$C_{1}$ & $\left\{I_{1}, I_{2}, I_{3}, I_{4}\right\}$ \\
$C_{2}$ & $\left\{I_{5}, I_{6}, I_{7}, I_{8}, I_{9}\right\}$ \\
$C_{3}$ & $\left\{I_{10}, I_{11}, I_{12}, I_{13}, I_{14}, I_{15}\right\}$ \\
$C_{4}$ & $\left\{I_{16}, I_{17}, I_{18}, I_{19}, I_{20}, I_{21}\right\}$ \\
$C_{5}$ & $\left\{I_{22}, I_{23}, I_{21}\right\}$ \\
$C_{6}$ & $\left\{I_{1}, I_{16}\right\}$ \\
$C_{7}$ & $\left\{I_{2}, I_{5}, I_{10}, I_{17}\right\}$ \\
$C_{8}$ & $\left\{I_{11}, I_{18}, I_{22}\right\}$ \\
$C_{9}$ & $\left\{I_{3}, I_{6}, I_{12}, I_{19}, I_{23}\right\}$ \\
$C_{10}$ & $\left\{I_{7}, I_{13}, I_{20}\right\}$ \\
$C_{11}$ & $\left\{I_{8}, I_{14}, I_{21}\right\}$ \\
$C_{12}$ & $\left\{I_{4}, I_{9}, I_{15}, I_{21}\right\}$ \\
\hline &
\end{tabular}

\subsection{Results and discussion}

\subsubsection{Moskowitz functions}

To evaluate the results of our nVT model, we extract the Moskowitz function at the upstream end of each link and compare it to the Moskowitz function derived from the SUMO output. Subsequently, we measure the average flow per cycle and calculate the differences between the results from nVT and SUMO. This results in a total of 43 (one per link) values for each demand scenario. Figure 12 shows the box-and-whisker diagrams for these differences related to each demand scenario.

On the left (Figure 12a) we show the absolute difference, and on the right (Figure 12b) the relative difference. The latter corresponds to the absolute difference divided by the average number of discharging vehicles per cycle according to SUMO. The absolute and relative errors are displayed on the y-axis, whereas the $\mathrm{x}$-axis corresponds to the volume-to-capacity ratio $V O C$. Generally, we observe very low errors. The relative error 


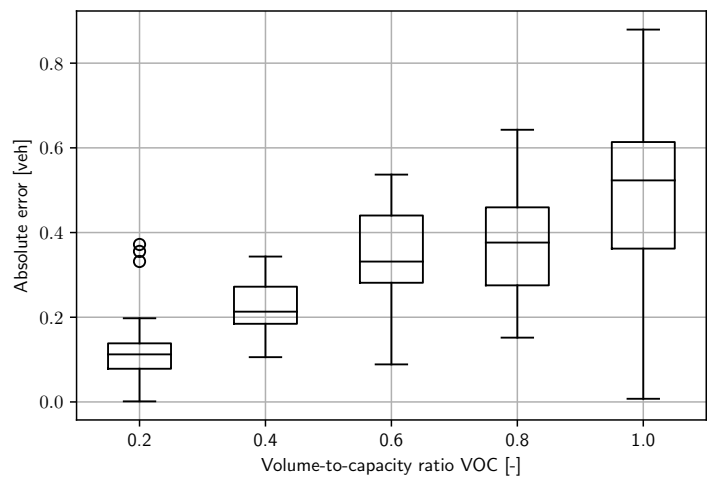

a) Absolute differences.

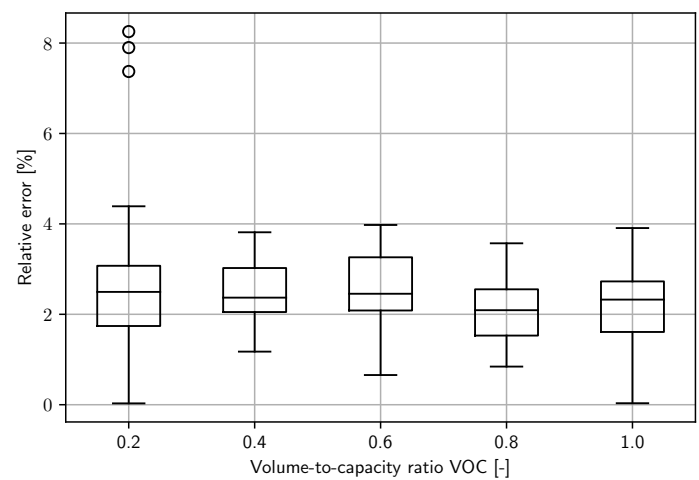

b) Relative differences.

Figure 12: Differences of cycle-based counts between nVT and SUMO for all demand scenarios.

for low demands is marginally higher, simply because of the low flows per cycle. Also, we can see that the median of the absolute error slightly increases with higher demands. This is clear, as a higher number of vehicles leads to more stochasticity and routing complexity in SUMO. This is also reflected by the growing inter-quartile range with growing demands. Most error observations are below $3 \%$ for all demand scenarios. For a $V O C=1$, this corresponds to an average difference of 0.5 vehicles counted per cycle in nVT and SUMO across all intersections. Given numerical errors, the above mentioned stochasticity, and the different nature of the microscopic and macroscopic modelling approaches, we adjudge this error as small.

In addition to these box-and-whisker diagrams, we present two Moskowitz functions in Figure 13 for illustration purposes. We display the Moskowitz functions $N$ from our nVT approach as solid black lines, and SUMO as dashed grey lines for the intersection $I_{3}$ (Figure 13a), and the inter-corridor connection $I_{18}$ (Figure 13b). Additionally, we plot the relative difference of both cumulative curves as dotted grey lines.

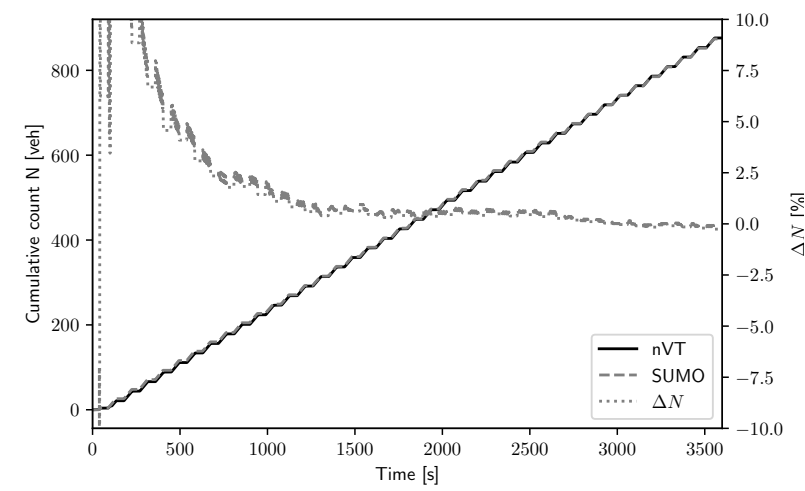

a) Moskowitz functions at intersection $I_{3}$.

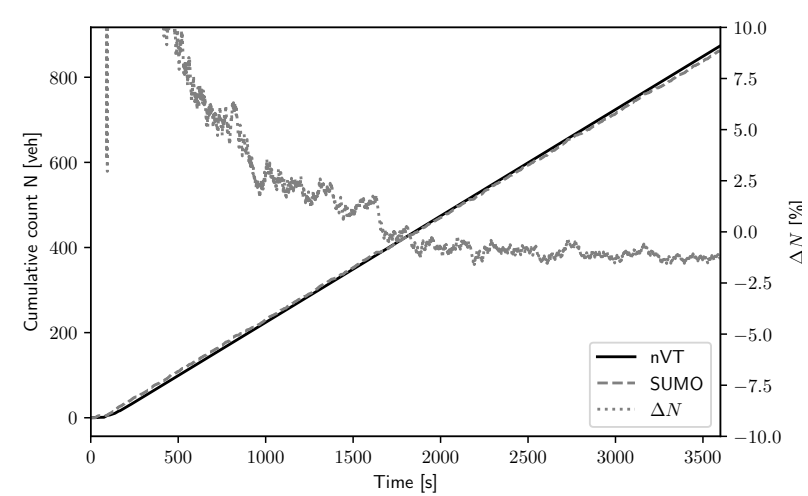

b) Moskowitz functions at inter-corridor connection $I_{18}$.

Figure 13: Moskowitz functions at an intersection and an inter-corridor connections from nVT and SUMO.

The left y-axis shows the Moskowitz functions $N$ in vehicles downstream of the intersections, while the right $\mathrm{y}$-axis shows the relative difference $\Delta N$ in per cent. The x-axis displays the simulation time in seconds. Figure 13a corresponds to an intersection, where there are no turning flows, i.e. $\alpha=0$. One can observe the characteristic step-wise increase due to the different signal phases. The relative difference is very high in the beginning, but soon converges to a value close to $0 \%$. The high values for low $t$ are due to the fact that the difference is related to the $N$ from SUMO, and these values are very low at the beginning of the simulation. Moreover, stochastic influences appear to be large for small simulation duration. However, after a reasonable 
simulation period (i.e. warm-up period), the results from SUMO and nVT match each other very well. Figure $13 \mathrm{~b}$ is an example for an inter-corridor connection. Due to the turning ratios $\alpha=0.5$, a constant flow occurs. Again, both curves match each other very well for longer simulation periods, although a minor difference of $1.5 \%$ remains after the simulation is finished. Note that this is the link with the largest difference in the whole network. Some of the reasons for these differences are mentioned in the paragraphs above. An in-depth sensitivity analysis (Ge et al., 2014) could be used to further explore these differences paying especial attention to the relation between the different inputs and the outputs of the SUMO simulations, but that's considered out of scope for this paper.

\subsubsection{Computational effort}

One great advantage of our method is the computational efficiency. This results from the macroscopic nature of the model. We show a comparison of the computational effort of both methods for the presented Sioux Falls case study in Table 2. The methods were evaluated on a computer with an Intel(R) Core(TM) i7-4600U CPU with $2.10 \mathrm{GHz}$ and $8 \mathrm{~GB}$ RAM.

Table 2: Computational effort comparison of SUMO and nVT

\begin{tabular}{lrrrr}
\hline$V O C$ & Computation time $[\mathrm{s}]$ & & \\
\hline & $\mathrm{SUMO}$ & $\mathrm{nVT}(\Delta t=0.1 \mathrm{~s})$ & $\mathrm{nVT}(\Delta t=1 \mathrm{~s})$ & $\mathrm{nVT}(\Delta t=5 \mathrm{~s})$ \\
\hline 0.2 & 10.1 & & & \\
0.4 & 17.2 & & & \\
0.6 & 28.2 & 70.5 & 3.5 & 0.6 \\
0.8 & 35.0 & & & \\
1.0 & 60.5 & & & \\
\hline
\end{tabular}

The demand scenarios are specified in the left-most column as a function of the $V O C$. We see that the computational cost in seconds for SUMO scales with the increasing demand. Since each vehicle is simulated separately it is logical that an increasing number of vehicles leads to an increasing computational effort. In contrast, this is not the case for $\mathrm{nVT}$ since the computational cost scales with the multi-dimensional variational graph $G$. The computational cost does not scale with demand, i.e. the actual number of vehicles simulated, but only with the number of points in $G$. The results in the table show the effect of an increased time-step $\Delta t$, which leads to a reduced number of points in $G$, and therefore to a drastic decrease of computational effort required. While $\Delta t=0.1 \mathrm{~s}$ leads to a high cost, the choice of $\Delta t=5 \mathrm{~s}$ results in a computational cost of two orders of magnitude lower than the highest one of SUMO. At the same time, no numerical error is introduced by the larger time-step as indicated in Section 4.2. The appropriate choice of the time-step makes our proposed nVT model suitable for real-time applications or as part of a model-based optimization framework.

Overall, this case study clearly shows the applicability of our nVT model at the network level. The results match those of a microscopic simulation for several demand scenarios very well. Moreover, the nVT framework can evaluate network-wide traffic dynamics at a low computational cost. We conclude that our nVT model represents a first step towards a fully VT-based KWT simulation applicable to large-scale networks.

\section{Conclusion}

This paper proposes an extension of the classical VT introduced by Daganzo (2005a) for the application at the network level. For this purpose, a network is decomposed into a set of non-overlapping corridors which include all edges. Subsequently, we propose a mathematical framework to incorporate real-valued source terms which are discrete in time and space into VT. This is necessary as flow conservation on corridors might not apply anymore. Our nVT model accounts for the corresponding discontinuities in the Moskowitz function and is able to propagate free-flow and congested traffic states across intersections and throughout the entire network. We successfully verify our framework for a simple network with the microscopic simulation SUMO. Additionally, we show that our extension inherits the numerical exact calculation of the Moskowitz function for triangular FDs from the original VT. Finally, we apply our framework to a case study for the Sioux Falls network. We again compare the results to the solution derived from SUMO. The traffic states predicted by our method clearly show a good fit to the ones from SUMO. Moreover, our results indicate the low computational cost which can be achieved with our VT extension. 
We conclude that we successfully extended VT for networks with real discrete source terms at intersections. For given origin flows and turning ratios, network-wide traffic conditions can be predicted according to KWT. This includes heterogeneous complex types of KWT problems, as time-space dependent FD and moving bottlenecks. The literature review showed the wide range of applications of VT, some of which may profit from the possibility to apply VT at the network level as well. For example, it opens the door to a network-wide evaluation of multi-modal traffic management strategies like the design and operation of intermittent bus lanes. Such a design can be formulated as a model-based optimization where nVT can effectively be applied as traffic simulation due to its low computational cost.

While our framework constitutes a clear contribution, many additions are relevant for future work. As we focused merely on signalized intersections, the implementation of merges seems to be the next logical step. One possibility could be the implementation of Daganzo's merging model into our model. This would additionally allow the modelling of partially conflicting traffic streams at intersections. Moreover, our framework is limited to fixed turning ratios. While this still allows varying transfer flows, stochastic turning ratios could also be considered in the future in order to enhance the model.

\section{Declaration of competing interests}

None.

\section{Acknowledgments}

Gabriel Tilg acknowledges the support by the research project MobiDig: Mobilität Digital Hochfranken funded by the German Federal Ministry of Transport and Digital Infrastructure. S. F. A. Batista and M. Menéndez acknowledge support by the NYUAD Center for Interacting Urban Networks (CITIES), funded by Tamkeen under the NYUAD Research Institute Award CG001 and by the Swiss Re Institute under the Quantum Cities $^{\text {TM }}$ initiative. Lukas Ambühl acknowledges the support by the ETH Research Grant ETH-27 16-1 under the project name SPEED.

\section{References}

Ambühl, L., Loder, A., Bliemer, M.C., Menendez, M., Axhausen, K.W., 2018. A functional form with a physical meaning for the macroscopic fundamental diagram. Transportation Research Part B: Methodological URL: http://www.sciencedirect.com/science/article/pii/S0191261517310123, doi:https://doi. org/10.1016/j.trb.2018.10.013.

Boyaci, B., Geroliminis, N., 2011. Estimation of the network capacity for multimodal urban systems. Procedia - Social and Behavioral Sciences 16, 803-813. doi:10.1016/j .sbspro.2011.04.499.

Chow, A.H., Li, S., Szeto, W., Wang, D.Z., 2015. Modelling urban traffic dynamics based upon the variational formulation of kinematic waves. Transportmetrica B: Transport Dynamics 3, 169-191.

Claudel, C.G., Bayen, A.M., 2010a. Lax-hopf based incorporation of internal boundary conditions into hamilton-jacobi equation. part i: Theory. IEEE Transactions on Automatic Control 55, $1142-1157$.

Claudel, C.G., Bayen, A.M., 2010b. Lax-hopf based incorporation of internal boundary conditions into hamilton-jacobi equation. part ii: Computational methods. IEEE Transactions on Automatic Control 55, 1158-1174.

Costeseque, G., Lebacque, J.P., 2014. A variational formulation for higher order macroscopic traffic flow models: Numerical investigation. Transportation Research Part B: Methodological 70,112 - 133. URL: http://www.sciencedirect.com/science/article/pii/S0191261514001477, doi:https: //doi.org/10.1016/j.trb.2014.08.012.

Daganzo, C., 1992. The cell transmission model. part i: A simple dynamic representation of highway traffic . Daganzo, C.F., 1995. The cell transmission model, part ii: network traffic. Transportation Research Part B: Methodological 29, 79-93.

Daganzo, C.F., 2005a. A variational formulation of kinematic waves: basic theory and complex boundary conditions. Transportation Research Part B: Methodological 39, 187-196. doi:10.1016/j.trb.2004.04. 003.

Daganzo, C.F., 2005b. A variational formulation of kinematic waves: Solution methods. Transportation Research Part B: Methodological 39, 934-950. doi:10.1016/j.trb.2004.05.003. 
Daganzo, C.F., Geroliminis, N., 2008. An analytical approximation for the macroscopic fundamental diagram of urban traffic. Transportation Research Part B: Methodological , 771-781doi:10.1016/j.trb.2008.06.008.

Daganzo, C.F., Knoop, V.L., 2016. Traffic flow on pedestrianized streets. Transportation Research Part B: Methodological 86, 211 - 222. URL: http://www.sciencedirect.com/science/article/pii/ S0191261516000023, doi:https://doi.org/10.1016/j.trb.2015.12.017.

Daganzo, C.F., Menendez, M., 2005. A variational formulation of kinematic waves: Bottleneck properties and examples, in: Proceedings of the 16th International Symposium on Transportation and Traffic Theory.

Dakic, I., Ambühl, L., Schümperlin, O., Menendez, M., 2020. On the modeling of passenger mobility for stochastic bi-modal urban corridors. Transportation Research Part C: Emerging Technologies 113, 146 163. doi:10.1016/j.trc.2019.05.018.

Duret, A., Yuan, Y., 2017. Traffic state estimation based on Eulerian and Lagrangian observations in a mesoscopic modeling framework. Transportation Research Part B: Methodological 101, 51-71. doi:10. 1016/j.trb.2017.02.008.

Friesz, T.L., Han, K., Neto, P.A., Meimand, A., Yao, T., 2013. Dynamic user equilibrium based on a hydrodynamic model. Transportation Research Part B: Methodological 47, 102-126.

Gayah, V.V., Ilgin Guler, S., Gu, W., 2016. On the impact of obstructions on the capacity of nearby signalised intersections. Transportmetrica B 4, 48-67. doi:10.1080/21680566.2015.1052111.

Ge, Q., Ciuffo, B., Menendez, M., 2014. An exploratory study of two efficient approaches for the sensitivity analysis of computationally expensive traffic simulation models. IEEE Transactions on Intelligent Transportation Systems 15, 1288-1297.

Girault, J.T., Gayah, V.V., Guler, I., Menendez, M., 2016. Exploratory analysis of signal coordination impacts on macroscopic fundamental diagram. Transportation Research Record: Journal of the Transportation Research Board , 36-46doi:10.3141/2560-05.

Guler, S.I., Gayah, V.V., Menendez, M., 2016. Bus priority at signalized intersections with single-lane approaches: A novel pre-signal strategy. Transportation Research Part C: Emerging Technologies 63, 51-70.

Han, K., Piccoli, B., Szeto, W., 2015. Continuous-time link-based kinematic wave model: formulation, solution existence, and well-posedness. Transportmetrica B: Transport Dynamics 4, 187-222.

Hans, E., Chiabaut, N., Leclercq, L., 2015. Applying variational theory to travel time estimation on urban arterials. Transportation Research Part B: Methodological 78, 169 - 181. URL: http://www. sciencedirect. com/science/article/pii/S019126151500079X, doi:https://doi.org/10.1016/j.trb.2015.04.004.

Jin, W.L., 2015. Continuous formulations and analytical properties of the link transmission model. Transportation Research Part B: Methodological 74, 88-103.

Kawai, K., Takenouchi, A., Ikawa, M., Kuwahara, M., 2019. Traffic state estimation using traffic measurement from the opposing lane - error analysis based on fluctuation of input data, in: Intelligent Transport Systems for Everyone's Mobility. Springer, pp. 247-263.

Kawasaki, Y., Hara, Y., Kuwahara, M., 2017. Real-time Monitoring of Dynamic Traffic States by State-Space Model. Transportation Research Procedia 21, 42-55. URL: http://dx.doi.org/10.1016/j.trpro. 2017. 03.076, doi:10.1016/j.trpro.2017.03.076.

Laval, J.A., Castrillón, F., 2015. Stochastic approximations for the macroscopic fundamental diagram of urban networks. Transportation Research Part B: Methodological 81, 904-916. doi:10.1016/j.trb.2015.09.002.

Laval, J.A., Costeseque, G., Chilukuri, B., 2016. The impact of source terms in the variational representation of traffic flow. Transportation Research Part B: Methodological 94, 204-216. doi:10.1016/j.trb. 2016. 09.011.

Laval, J.A., Leclercq, L., 2013. The Hamilton-Jacobi partial differential equation and the three representations of traffic flow. Transportation Research Part B: Methodological 52, 17-30. URL: http://dx.doi.org/10. 1016/j.trb.2013.02.008, doi:10.1016/j.trb.2013.02.008.

Lebacque, J.P., Khoshyaran, M.M., 2013. A variational formulation for higher order macroscopic traffic flow models of the GSOM family. Transportation Research Part B: Methodological 57, 245-265. URL: http://dx.doi.org/10.1016/j.trb.2013.07.005, doi:10.1016/j.trb.2013.07.005.

Leclercq, L., Geroliminis, N., 2013. Estimating mfds in simple networks with route choice. Transportation Research Part B Methodological 57, 468-484. doi:10.1016/j.trb.2013.05.005. 
Leclercq, L., Laval, J., Chevallier, E., 2007. The lagrangian coordinate system and what it means for first order traffic flow model, the proceedings of the 17th international symposium on transportation and traffic theory.

Leclercq, L., Paipuri, M., 2019. Macroscopic Traffic Dynamics Under Fast-Varying Demand. Transportation Science 53, 1526-1545. doi:10.1287/trsc.2019.0908.

Li, J., Zhang, H.M., 2015. Bounding tandem queuing system performance with variational theory. Transportation Research Part B: Methodological 81, 848-862. URL: http://dx.doi.org/10.1016/j.trb.2015.07. 013, doi:10.1016/j.trb.2015.07.013.

Lighthill, M.J., Whitham, G.B., 1955. On kinematic waves ii. a theory of traffic flow on long crowded roads. Proc. R. Soc. Lond. A 229, 317-345.

Loder, A., Dakic, I., Bressan, L., Ambühl, L., Bliemer, M.C., Menendez, M., Axhausen, K.W., 2019. Capturing network properties with a functional form for the multi-modal macroscopic fundamental diagram. Transportation Research Part B: Methodological 129, 1-19.

Lopez, P.A., Behrisch, M., Bieker-Walz, L., Erdmann, J., Flötteröd, Y.P., Hilbrich, R., Lücken, L., Rummel, J., Wagner, P., Wießner, E., 2018. Microscopic traffic simulation using sumo, in: The 21st IEEE International Conference on Intelligent Transportation Systems, IEEE.

Mazaré, P.E., Dehwah, A.H., Claudel, C.G., Bayen, A.M., 2011. Analytical and grid-free solutions to the Lighthill-Whitham-Richards traffic flow model. Transportation Research Part B: Methodological 45, 1727-1748. doi:10.1016/j.trb.2011.07.004.

Mehran, B., Kuwahara, M., 2013. Fusion of probe and fixed sensor data for short-term traffic prediction in urban signalized arterials. International Journal of Urban Sciences 17, 163-183. doi:10.1080/12265934. 2013.776291.

Mehran, B., Kuwahara, M., Naznin, F., 2012. Implementing kinematic wave theory to reconstruct vehicle trajectories from fixed and probe sensor data. Transportation research part C: emerging technologies 20, $144-163$.

Moskowitz, K., 1965. Discussion of 'freeway level of service as influenced by volume and capacity characteristics' by d.r. drewand c. j. keese. Highway Research Record 99, 43-44.

Newell, G.F., 1993. A simplified theory of kinematic waves in highway traffic, part i: General theory, part ii: Queueing at freeway bottlenecks, part iii: Multi-destination flows. Transportation Research Part B: Methodological 27, 281-313.

Richards, P.I., 1956. Shock waves on the highway. Operations research 4, 42-51.

Saeednia, M., Menendez, M., 2016. A decision support system for real-time platooning of trucks, in: 2016 IEEE 19th International Conference on Intelligent Transportation Systems (ITSC), IEEE. pp. 1792-1797.

Seo, T., Bayen, A.M., Kusakabe, T., Asakura, Y., 2017. Traffic state estimation on highway: A comprehensive survey. Annual Reviews in Control 43, 128-151.

Sun, Z., Ban, X.J., 2013. Vehicle trajectory reconstruction for signalized intersections using mobile traffic sensors. Transportation Research Part C: Emerging Technologies 36, 268-283.

Takenouchi, A., Kawai, K., Kuwahara, M., 2019. Traffic state estimation and its sensitivity utilizing measurements from the opposite lane. Transportation Research Part C: Emerging Technologies 104, 95-109. URL: https://doi.org/10.1016/j.trc.2019.04.016, doi:10.1016/j.trc.2019.04.016.

Tampère, C.M., Corthout, R., Cattrysse, D., Immers, L.H., 2011. A generic class of first order node models for dynamic macroscopic simulation of traffic flows. Transportation Research Part B: Methodological 45, 289-309. doi:10.1016/j.trb.2010.06.004.

Tilg, G., Amini, S., Busch, F., 2020. Evaluation of analytical approximation methods for the macroscopic fundamental diagram. Transportation Research Part C: Emerging Technologies 114, 1-19. URL: http://www.sciencedirect.com/science/article/pii/S0968090X19307661, doi:https://doi. org $/ 10.1016 / j . \operatorname{trc} .2020 .02 .003$.

Wada, K., Usui, K., Takigawa, T., Kuwahara, M., 2018. An optimization modeling of coordinated traffic signal control based on the variational theory and its stochastic extension. Transportation Research Part B: Methodological 117, 907 - 925. URL: http://www.sciencedirect.com/science/article/pii/ S019126151730749X, doi:https://doi.org/10.1016/j.trb.2017.08.031. tRB:ISTTT-22. 
Wu, K., Guler, S.I., Gayah, V.V., 2017. Estimating the impacts of bus stops and transit signal priority on intersection operations: Queuing and variational theory approach. Transportation Research Record 2622, 70-83. doi:10.3141/2622-07.

Yperman, I., 2007. The link transmission model for dynamic network loading . 